\title{
A Method for Calculating Export Supply and Import Demand Elasticities
}

Stephen Tokarick 


\title{
IMF Working Paper
}

Research Department

\section{A Method for Calculating Export Supply and Import Demand Elasticities}

\author{
Prepared by Stephen Tokarick*
}

Authorized for distribution by Andrew Berg

July 2010

\begin{abstract}

\section{This Working Paper should not be reported as representing the views of the IMF.} The views expressed in this Working Paper are those of the author(s) and do not necessarily represent those of the IMF or IMF policy. Working Papers describe research in progress by the author(s) and are published to elicit comments and to further debate.

Trade elasticities are often needed in applied country work for various purposes and this paper describes a method for estimating import demand and export supply elasticities without using econometrics. The paper reports empirical estimates of these elasticities for a large number of low, middle, and upper income countries. One task for which trade elasticities are needed is in developing exchange rate assessments and this paper shows how the estimated elasticities can be used for this purpose.
\end{abstract}

JEL Classification Numbers:F11, F32

Keywords:import, export, elasticities

Author’s E-Mail Address: STokarick@imf.org

\footnotetext{
* This paper is part of the work on a larger project assessing exchange rates for low-income countries. I thank members of the team, Alessandro Prati, Luca Ricci, Thierry Tressel, Oya Celasun, and Lone Christiansen for helpful comments. Research assistance by Freddy Cama and Manzoor Gill is gratefully acknowledged.
} 
I. Introduction 3

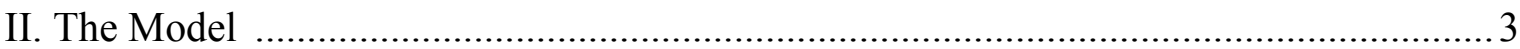

A. Assumptions ......................................................................................... 3

B. Model Solution .......................................................................................... 4

C. Sensitivity of Elasticities to Parameter Values ................................................... 7

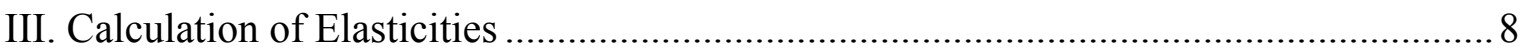

IV. Computing Elasticities of a Country's Trade Balance With Respect to a Change in the Exchange Rate ..................................................................................................... 22

A. The Trade Balance and the Real Exchange Rate .........................................22

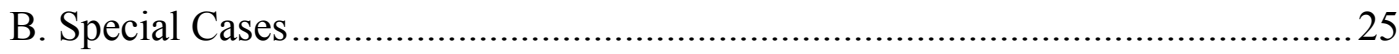

C. Price Elasticities and the Real Exchange Rate ............................................... 35

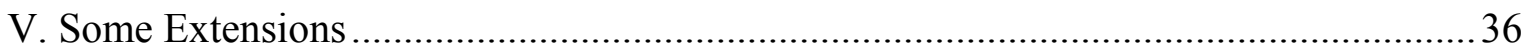

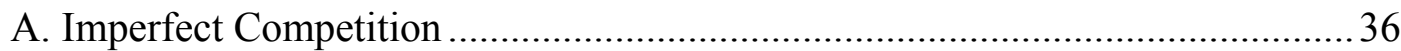

B. Less than Perfect Labor Mobility ................................................................. 37

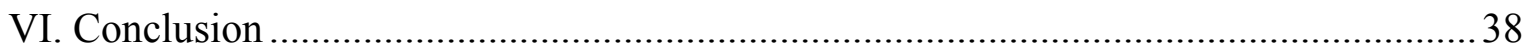

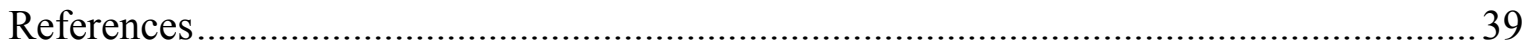

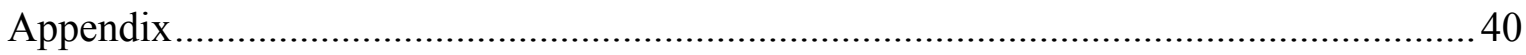

Tables

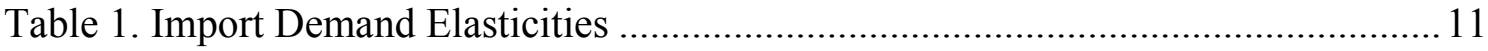

Table 2. Export Supply Elasticities........................................................................... 17

Table 3. Summary of How Changes in the Exchange Rate Affect the Trade Balance... 27

Table 4. Trade Balance Elasticities. 


\section{INTRODUCTION}

This paper sets out the details of a methodology that can be used to calculate export supply and import demand elasticities without using econometrics. There is a vast literature (see Stern, Francis, and Schmacher (1976) and Khan and Goldstein (1985) for surveys) that contains empirical estimates of trade elasticities, but the magnitude of the estimates varies widely, and in some instances, the signs of the estimates are contrary to theory. The methodology presented below uses a well-accepted model of international trade, together with a comprehensive dataset, to calculate elasticity values.

Export supply and import demand elasticities can be calculated by using some results from production theory. For example, it is well-known that the derivative of an economy's GDP function with respect to an output price gives the general-equilibrium supply function, using Hotelling's lemma. The demand function for inputs can be obtained in a similar fashion and this approach has been used by Kee et al. (2008) and others to estimate import demand elasticities. More generally, there is a large literature in international trade which uses the GDP function approach to estimate elasticities (see for example Kohli (1991)). In this approach, the demand for imports arises from the demand for imported intermediate inputs. Even if one assumes that there is a demand for imports for consumption, this approach is still valid if one assumes that imports for consumption arise in the production sector, since they have to be combined with wholesaling and retail services before they are consumed. In this sense, even imports for consumption can be thought of as an intermediate input.

\section{THE MODEL}

\section{A. Assumptions}

The methodology uses a standard general equilibrium model from international trade theory, as described in Jones (1965), Dixit and Norman (1980) and Woodland (1982). The model assumes that an economy produces three goods (i) a good which is exported, denoted by (E); (ii) a good which competes with imports, denoted by (M); and (iii) a nontraded good (N). It is assumed that there is no local demand for the exportable good. Each of these three goods is produced using labor (L) which is mobile across sectors; a factor specific to each sector (K); and imported intermediate inputs (I). Since labor is assumed to be mobile across sectors, it must earn the same wage regardless of where it is employed. The return to the specific factor in each sector will, of course, differ. The price of imported intermediates is assumed to be exogenous. The output prices of all three goods are treated as parameters.

It is assumed that the output of each good is produced under constant returns to scale and zero profits. Therefore, the following conditions must hold: 


$$
\begin{gathered}
w a_{L E}+r_{E} a_{K E}+p_{I} a_{I E}=p_{E} \\
w a_{L M}+r_{M} a_{K M}+p_{I} a_{I M}=p_{M} \\
w a_{L N}+r_{N} a_{K N}+p_{I} a_{I N}=p_{N}
\end{gathered}
$$

where $p_{E}$ is the domestic price of exports, $p_{M}$ is the domestic price of imports (inclusive of any tariff), $p_{N}$ is the price of the nontraded good, $a_{i j}$ is the amount of factor $\mathrm{i}$ ( $\mathrm{i}=$ labor, capital, imported inputs) used per unit of good $\mathrm{j}, w$ is the wage rate, $r_{j}$ is the return to capital in sector $\mathrm{j}$, and $p_{I}$ is the exogenously given price of imported intermediate inputs.

The primary factors of production — labor and capital — are assumed to be fully employed:

$$
\begin{gathered}
a_{K E} X_{E}=K_{E} \\
a_{K M} X_{M}=K_{M} \\
a_{K N} X_{N}=K_{N} \\
a_{L E} X_{E}+a_{L M} X_{M}+a_{L N} X_{N}=L
\end{gathered}
$$

where $K_{j}$ is the amount of capital used in sector $\mathrm{j}, L$ is the endowment of labor in the economy, and $X_{j}$ is output of good $\mathrm{j}$.

Equations (1) through (3) reflect the assumption that the price of each good must equal per unit cost. That is, per-unit labor costs, plus per-unit capital costs, plus the per-unit cost of imported inputs must equal the output price of each good. This zero-profit condition implicitly assumes perfect competition. A subsequent section discusses how imperfect competition could be introduced. Equations (4) through (6) represent the assumption that capital is sector specific, reflecting a short to medium-run focus. Equation (7) requires that the labor market clear: the amount of labor used in each sector must equal the economy-wide endowment.

\section{B. Model Solution}

Totally differentiating equations (1) through (7) and putting them in proportional change form gives:

$$
\begin{gathered}
\hat{w} \theta_{L E}+\hat{r}_{E} \theta_{K E}+\hat{p}_{I} \theta_{I E}=\hat{p}_{E} \\
\hat{w} \theta_{L M}+\hat{r}_{M} \theta_{K M}+\hat{p}_{I} \theta_{I M}=\hat{p}_{M}
\end{gathered}
$$




$$
\begin{gathered}
\hat{w} \theta_{L N}+\hat{r}_{N} \theta_{K N}+\hat{p}_{I} \theta_{I N}=\hat{p}_{N} \\
\lambda_{K E} \hat{X}_{E}=\hat{K}_{E}-\lambda_{K E} \hat{a}_{K E} \\
\lambda_{K M} \hat{X}_{M}=\hat{K}_{M}-\lambda_{K M} \hat{a}_{K M} \\
\lambda_{K N} \hat{X}_{N}=\hat{K}_{N}-\lambda_{K N} \hat{a}_{K N} \\
\lambda_{L E} \hat{X}_{E}+\lambda_{L M} \hat{X}_{M}+\lambda_{L N} \hat{X}_{N}=\hat{L}-\hat{a}_{L E} \lambda_{L E}-\hat{a}_{L M} \lambda_{L M}-\hat{a}_{L N} \lambda_{L N}
\end{gathered}
$$

In the above equations, $\theta_{i j}$ is the share of good $\mathrm{j}$ 's cost accounted for by factor $\mathrm{i}, \lambda_{i j}$ is the proportion of the supply of factor $\mathrm{i}$ used in industry $\mathrm{j}$, and a “^” denotes proportional change, e.g., $\hat{p}=\frac{d p}{p}$. As a result of the assumed structure, the following relationships must hold:

$$
\begin{aligned}
& \text { 1. } \sum_{i} \theta_{i j}=1 \text {, for each sector } \mathrm{j} \text {; } \\
& \text { 2. } \sum_{j} \lambda_{i j}=1 \text {, for each factor } \mathrm{i} \text {. }
\end{aligned}
$$

Each $a_{i j}$, the factor demands per unit of output, depends on the input prices:

$$
a_{i j}=a_{i j}\left(w, r_{j}, p_{I}\right)
$$

Each $a_{i j}$ can also be related to the elasticity of substitution between labor, capital, and imported inputs in each sector $\mathrm{j}$. Assuming that the elasticity of substitution among all three factors is the same, the following relationships hold for each sector $\mathrm{j}$, using the definition of the elasticity of substitution, $\sigma_{j}$ :

$$
\sigma_{j}=\frac{\hat{a}_{K j}-\hat{a}_{L j}}{\hat{w}-\hat{r}_{j}}, \text { or } \quad \sigma_{j}\left(\hat{w}-\hat{r}_{j}\right)=\hat{a}_{K j}-\hat{a}_{L j} \text {. }
$$

and:

$$
\sigma_{j}=\frac{\hat{a}_{K j}-\hat{a}_{I j}}{\hat{p}_{I}-\hat{r}_{K j}}, \text { or } \quad \sigma_{j}\left(\hat{p}_{I}-\hat{r}_{K j}\right)=\hat{a}_{K j}-\hat{a}_{I j} \text {. }
$$

and: 


$$
\sigma_{j}=\frac{\hat{a}_{L j}-\hat{a}_{I j}}{\hat{p}_{I}-\hat{w}}, \text { or } \quad \sigma_{j}\left(\hat{p}_{I}-\hat{w}\right)=\hat{a}_{L j}-\hat{a}_{I j}
$$

Cost minimization requires that:

$$
\begin{gathered}
\theta_{L E} \hat{a}_{L E}+\theta_{K E} \hat{a}_{K E}+\theta_{I E} \hat{a}_{I E}=0 \\
\theta_{L M} \hat{a}_{L M}+\theta_{K M} \hat{a}_{K M}+\theta_{I M} \hat{a}_{I M}=0 \\
\theta_{L N} \hat{a}_{L N}+\theta_{K N} \hat{a}_{K N}+\theta_{I N} \hat{a}_{I N}=0
\end{gathered}
$$

Equations (17) through (22) can be used to solve for each $\hat{a}_{i j}$, as function of the factor prices, the elasticity of substitution between labor, capital, and imported inputs in each sector, and the relevant cost shares (see Jones (1965)). Using the above relationships, the solutions for each $\hat{a}_{i j}$ are:

$$
\begin{gathered}
\hat{a}_{L E}=-\sigma_{E}\left(\hat{w}-\hat{r}_{E}\right) \theta_{K E}-\theta_{I E} \sigma_{E}\left(\hat{w}-\hat{p}_{I}\right) \\
\hat{a}_{K E}=\sigma_{E}\left(\hat{w}-\hat{r}_{E}\right) \theta_{L E}+\theta_{I E} \sigma_{E}\left(\hat{p}_{I}-\hat{r}_{E}\right) \\
\hat{a}_{I E}=\sigma_{E}\left(\hat{w}-\hat{p}_{I}\right) \theta_{L E}+\theta_{K E} \sigma_{E}\left(\hat{r}_{E}-\hat{p}_{I}\right) \\
\hat{a}_{L M}=-\sigma_{M}\left(\hat{w}-\hat{r}_{M}\right) \theta_{K M}-\theta_{I M} \sigma_{M}\left(\hat{w}-\hat{p}_{I}\right) \\
\hat{a}_{K M}=\sigma_{M}\left(\hat{w}-\hat{r}_{M}\right) \theta_{L M}+\theta_{I M} \sigma_{M}\left(\hat{p}_{I}-\hat{r}_{M}\right) \\
\hat{a}_{I M}=\sigma_{M}\left(\hat{w}-\hat{p}_{I}\right) \theta_{L M}+\theta_{K M} \sigma_{M}\left(\hat{r}_{M}-\hat{p}_{I}\right) \\
\hat{a}_{L N}=-\sigma_{N}\left(\hat{w}-\hat{r}_{N}\right) \theta_{K N}-\theta_{I N} \sigma_{N}\left(\hat{w}-\hat{p}_{I}\right) \\
\hat{a}_{K N}=\sigma_{N}\left(\hat{w}-\hat{r}_{N}\right) \theta_{L N}+\theta_{I N} \sigma_{N}\left(\hat{p}_{I}-\hat{r}_{N}\right) \\
\hat{a}_{I N}=\sigma_{N}\left(\hat{w}-\hat{p}_{I}\right) \theta_{L N}+\theta_{K N} \sigma_{N}\left(\hat{r}_{N}-\hat{p}_{I}\right)
\end{gathered}
$$

Equations (23) through (31) show how each factor demand (per unit of output) responds to changes in input prices.

Substituting equations (23) through (31) into equations (8) through (14), it is possible to solve for all the endogenous variables $\left(\hat{w}, \hat{r}_{E}, \hat{r}_{M}, \hat{r}_{N}, \hat{X}_{E}, \hat{X}_{M} \hat{X}_{N}\right)$, as a function of the exogenous variables $\left(\hat{L}, \hat{K}_{E} \hat{K}_{M} \hat{K}_{N}, \hat{p}_{E}, \hat{p}_{M}, \hat{p}_{N}, \hat{p}_{I}\right)$. 
Since the objective is to determine values for the export supply elasticity and the import demand elasticity, two relationships are of interest:

1. Export supply elasticity $=\frac{\hat{X}_{E}}{\hat{p}_{E}}$, which can be obtained from the equation for the output of the exportable good: $\hat{X}_{E}=F\left(\hat{L}, \hat{K}_{E}, \hat{K}_{M}, \hat{K}_{N}, \hat{p}_{E}, \hat{p}_{M}, \hat{p}_{I}\right)$. The coefficient of the term $\hat{p}_{E}$ gives the export supply elasticity, which is:

$\frac{\hat{X}_{E}}{\hat{p}_{E}}=\frac{\lambda_{L E} \sigma_{E} \theta_{K M} \theta_{K N} \theta_{I E} \sigma_{E}+\lambda_{L M} \sigma_{M} \theta_{K N}\left(1-\theta_{I M}\right) \sigma_{E}\left(1-\theta_{K E}\right)+\lambda_{L N} \sigma_{N} \theta_{K M}\left(1-\theta_{I N}\right) \sigma_{E}\left(1-\theta_{K E}\right)}{\lambda_{L E} \sigma_{E} \theta_{K M} \theta_{K N}\left(1-\theta_{I E}\right)+\lambda_{L M} \sigma_{M} \theta_{K E} \theta_{K N}\left(1-\theta_{I M}\right)+\lambda_{L N} \sigma_{N} \theta_{K E} \theta_{K M}\left(1-\theta_{I N}\right)}$

2. Import demand arises from the demand for imported intermediate inputs. Total demand for imported inputs in the economy $\left(M_{I}\right)$ is:

$M_{I}=\sum_{j} M_{I j}$, where $M_{I j}=a_{I j} X_{j}$. Therefore,

$\hat{M}_{I}=\lambda_{I E} \hat{M}_{I E}+\lambda_{I M} \hat{M}_{I M}+\lambda_{I N} \hat{M}_{I N}$, and $\hat{M}_{I j}=\hat{a}_{I j}+\hat{X}_{j}$

Using the solutions for $\hat{a}_{I j}$ and $\hat{X}_{j}$, it is possible to solve for $\hat{M}_{I}$ as a function of $\hat{p}_{I}$.

Therefore, the import demand elasticity $=\frac{\hat{M}_{I}}{\hat{p}_{I}}$, which equals:

$$
\begin{aligned}
& \frac{\hat{M}_{I}}{\hat{p}_{I}}=\frac{1}{\left(\lambda_{L E} \sigma_{E} \theta_{K M} \theta_{K N}\left(1-\theta_{I E}\right)+\lambda_{L M} \sigma_{M} \theta_{K E} \theta_{K N}\left(1-\theta_{I M}\right)+\lambda_{L N} \sigma_{N} \theta_{K E} \theta_{K M}\left(1-\theta_{I N}\right)\right.} \\
& {\left[\begin{array}{l}
-\lambda_{I E}\left[\sigma_{E} \lambda_{L E} \sigma_{E} \theta_{K M} \theta_{K N}+\sigma_{E} \lambda_{L M} \sigma_{M} \theta_{K N}\left(1-\theta_{I M}-\theta_{L E}\right)+\sigma_{E} \lambda_{L N} \sigma_{N} \theta_{K M}\left(1-\theta_{I N}-\theta_{L E}\right)\right] \\
-\lambda_{I M}\left[\sigma_{M} \lambda_{L E} \sigma_{E} \theta_{K N}\left(1-\theta_{I E}-\theta_{L M}\right)+\sigma_{M} \lambda_{L M} \sigma_{M} \theta_{K E} \theta_{K N}+\sigma_{M} \lambda_{L N} \sigma_{N} \theta_{K E}\left(1-\theta_{I N}-\theta_{L M}\right)\right] \\
-\lambda_{I N}\left[\sigma_{N} \lambda_{L E} \sigma_{E} \theta_{K M}\left(1-\theta_{I E}-\theta_{L N}\right)+\sigma_{N} \lambda_{L M} \sigma_{M} \theta_{K E}\left(1-\theta_{I M}-\theta_{L N}\right)+\sigma_{N} \lambda_{L N} \sigma_{N} \theta_{K E} \theta_{K M}\right]
\end{array}\right]}
\end{aligned}
$$

\section{Sensitivity of Elasticities to Parameter Values}

This section discusses how the calculated elasticities are affected by the underlying parameters: (i) the elasticity of substitution between factors $\left(\sigma_{j}\right)$; (ii) the cost share of factor $\mathrm{i}$ in the production of good $\mathrm{j}\left(\theta_{i j}\right)$; and (iii) the proportion of the total supply of factor $\mathrm{i}$ used in the production of good $\mathrm{j}\left(\lambda_{i j}\right)$. 
- $\quad$ Changes in the elasticities of substitution between factors $\left(\sigma_{j}\right)$ : Increases in $\sigma_{j}$ will increase the magnitude of both the export supply and import demand elasticity, regardless of the sector. The reason for this is that a higher value for $\sigma_{j}$ makes it easier to alter factor proportions, i.e. factor usage, in each sector. Therefore, regarding export supply, a higher $\sigma_{j}$ will make it easier to increase output and will therefore increase the export supply elasticity. Regarding the demand for imported intermediate inputs, a higher value for $\sigma_{j}$ will make it easier for firms to substitute between labor and imported intermediates. Firms can alter factor usage more easily; therefore the import demand elasticity will be larger.

- Changes in the factor cost shares, $\left(\theta_{i j}\right)$ : Under model assumptions, capital is assumed to be fixed by sector, while labor can move freely across sectors. Similarly, firms can freely alter the amounts of imported intermediate inputs they use. Therefore, larger values for $\left(\theta_{L j}\right)$ and $\left(\theta_{I j}\right)$, and thus smaller values for $\left(\theta_{K j}\right)$, will increase the magnitude of the export supply elasticity. The larger is $\theta_{K j}$, the more difficult it will be for firms to increase output in response to a price change because capital stocks are fixed by sector.

- $\quad$ Changes in the distributive shares, $\left(\lambda_{i j}\right)$ : The effect of changes in the distributive shares can either raise or lower the magnitudes of the elasticities.

\section{Calculation of Elasticities}

Equations (32) and (33) give the elasticities of interest and can be calculated for values of $\sigma_{j}, \theta_{i j}$, and $\lambda_{i j}$. This section explains how values for these parameters can be obtained.

Data on $\theta_{i j}$, and $\lambda_{i j}$ for 87 countries/regions are available from the Global Trade Analysis Project (GTAP) database for the years 1997, and 2001. Data are available for 2004 for 113 countries/regions. The countries/regions in the database include a mix of developed (24) and developing countries (63). This database contains information on value added by sector, as well as its components (primary inputs), since it is based on country input-output tables. The GTAP database is described in Dimaranan and McDougal (2006).

Developed Countries: Australia, New Zealand, China, Hong Kong, Japan, Korea, Canada, United States, United Kingdom, Austria, Belgium, Denmark, Finland, France, Germany, Greece, Ireland, Italy, Luxembourg, Netherlands, Portugal, Spain, Sweden, Switzerland.

Developing Countries: Rest of countries in Oceania, Taiwan, Rest of East Asia, Indonesia, Malaysia, Philippines, Singapore, Thailand, Vietnam, Rest of Southeast Asia, Bangladesh, India, Sri Lanka, Rest of South Asia, Mexico, Rest of North America, Columbia, Peru, 
Venezuela, Andean Pact countries, Argentina, Brazil, Chile, Uruguay, Rest of South America, Central America, Countries of the Caribbean, Countries of the free trade area of the Americas, Rest of European Free Trade Area, Rest of Europe, Albania, Bulgaria, Croatia, Cyprus, Czech Republic, Hungary, Malta, Poland, Romania, Slovak Republic, Slovenia, Estonia, Latvia, Lithuania, Russia, Rest of former Soviet Union, Turkey, Rest of the Middle East, Morocco, Tunisia, Rest of North Africa, Botswana, South Africa, Rest of South African Customs Union, Malawi, Mozambique, Tanzania, Zambia, Zimbabwe, Madagascar, Uganda,

Rest of South African Development Community, Rest of sub-Saharan Africa.

To apply the methodology described above, the following strategy is adopted:

- $\quad$ The GTAP database contains data on labor, capital, and imported inputs for 57 sectors. Starting from the full database, the 57 sectors in each country were aggregated into 3 sectors per country (exportables, importables, and nontraded) using sectoral data on trade flows. If exports or imports from a sector was 10 percent of value added or less, the sector was classified as nontradeable. A sector was considered exportable if exports exceeded imports and exports exceeded 10 percent of value added. A sector was importable otherwise.

- $\quad$ The GTAP database contains a value for the elasticity of substitution among factors used in the 57 sectors for each country. The values are taken from various econometric studies. It assumes that these elasticities are the same for all 87 countries and regions. The elasticities are aggregated from the 57 sectors into 3, using data on value-added shares in each sector.

- $\quad$ Once the sectors were classified into 3 categories, $\lambda_{i j}$ and $\theta_{i j}$ were calculated for each country. Then, using the elasticities of substitution, equations (32) and (33) were used to calculate the elasticities.

Import demand and export supply elasticities are calculated using the procedure described above. There are several "types" of elasticities:

- Both a "short run" and a "long run" import demand and export supply elasticity were calculated. The short-run elasticities correspond to a set of short-run elasticities of substitution among inputs (sigmas), while the long-run elasticities correspond to a long-run set of sigmas. In general, the long-run sigmas are higher in magnitude, compared to the short-run sigmas.

- Import demand and export supply elasticities are calculated for two sets of assumptions: (i) elasticities are computed with respect to their own price (the standard definition of elasticities); and (ii) including general equilibrium effects, where the latter takes into account changes in both the own price and the price of other traded goods. For example, the own export supply elasticity measures how export supply 
changes as the price of exports changes, holding all other prices constant. A devaluation for example increases the prices of imported intermediate inputs, which will reduce the export supply response to the extent that exports use imported inputs. The mathematical formulas for the "general equilibrium" elasticities are shown in the appendix.

Elasticity values are reported in the following tables:

Table 1: Import demand elasticities. This table reports estimated import demand elasticities from various studies, as well as from the method described above. The study labelled "World Bank" refers to a study conducted by Kee, Nicita, and Olarreaga (2008). In that study, the authors estimated import demand elasticities, using the GDP function approach, which is similar to the one used in this paper. The study labelled "Senhadji" refers to a study conducted by Senhadji (1998), who estimated import demand elasticities using what might be termed the "traditional" approach: regressing imports on relative prices and real income. GTAP 2001 refers to the data from version 6 of the GTAP database (data for the year 2001), while GTAP 2004 refers to data from version 7 of the GTAP database (data for the year 2004). For each of these two datasets, a set of "short-run"(SR) and "long-run" (LR) elasticities are reported. The short run is defined to be about six months to a year at the most, while long run refers to as many as three years or more. As well, a set of both short-run and long-run elasticities are reported that take into account general equilibrium effects. The columns on the far right of the table show the average elasticities calculated for 2001 and 2004. 
Table 1. Import Demand Elasticities

GTAP2001 GTAP2004

\begin{tabular}{|c|c|c|c|c|c|c|c|c|c|c|c|c|c|c|}
\hline & \multirow[b]{3}{*}{ Kee et al. (2008) } & \multirow[b]{3}{*}{ Senhadji (1997) } & \multicolumn{4}{|c|}{ GTAP2001 } & \multicolumn{4}{|c|}{ GTAP2004 } & \multicolumn{4}{|c|}{ Average 2001-2004 } \\
\hline & & & \multirow[b]{2}{*}{ SR } & \multirow[b]{2}{*}{ LR } & \multicolumn{2}{|c|}{$\begin{array}{c}\text { Adjusted to include GE } \\
\text { effects }\end{array}$} & \multirow[b]{2}{*}{ SR } & \multirow[b]{2}{*}{ LR } & \multicolumn{2}{|c|}{$\begin{array}{c}\text { Adjusted to include GE } \\
\text { effects }\end{array}$} & \multirow[b]{2}{*}{ SR } & \multirow[b]{2}{*}{ LR } & \multicolumn{2}{|c|}{$\begin{array}{c}\text { Adjusted to include GE } \\
\text { effects }\end{array}$} \\
\hline & & & & & SR & LR & & & SR & LR & & & SR & LR \\
\hline \multicolumn{15}{|l|}{ Low income } \\
\hline Bangladesh & -1.61 & & -1.23 & -1.69 & -0.25 & -0.33 & -1.24 & -1.71 & -0.24 & -0.33 & -1.23 & -1.70 & -0.24 & -0.33 \\
\hline Benin & -1.08 & -0.54 & -0.99 & -1.33 & -0.95 & -1.29 & -1.32 & -1.77 & -1.12 & -1.53 & -1.15 & -1.55 & -1.04 & -1.41 \\
\hline Burkina Faso & -1.06 & & -0.99 & -1.33 & -0.95 & -1.29 & -1.32 & -1.77 & -1.12 & -1.53 & -1.15 & -1.55 & -1.04 & -1.41 \\
\hline Burundi & -1.1 & -0.27 & -0.99 & -1.33 & -0.95 & -1.29 & -0.99 & -1.33 & -0.87 & -1.18 & -0.99 & -1.33 & -0.91 & -1.23 \\
\hline Cambodia & & & -0.97 & -1.33 & -0.83 & -1.15 & -1.82 & -2.54 & -1.05 & -1.51 & -1.39 & -1.93 & -0.94 & -1.33 \\
\hline Central African Rep. & -1.04 & & -0.99 & -1.33 & -0.95 & -1.29 & -1.02 & -1.40 & -1.03 & -1.41 & -1.01 & -1.37 & -0.99 & -1.35 \\
\hline Chad & -1.02 & & -0.99 & -1.33 & -0.95 & -1.29 & -1.02 & -1.40 & -1.03 & -1.41 & -1.01 & -1.37 & -0.99 & -1.35 \\
\hline Congo, Dem. Rep. & & & -0.93 & -1.25 & -0.60 & -0.83 & -1.07 & -1.45 & -1.00 & -1.37 & -1.00 & -1.35 & -0.80 & -1.10 \\
\hline Comoros & -1.08 & & -0.99 & -1.33 & -0.95 & -1.29 & -0.99 & -1.33 & -0.87 & -1.18 & -0.99 & -1.33 & -0.91 & -1.23 \\
\hline Côte d'Ivoire & -1.12 & -0.46 & -0.99 & -1.33 & -0.95 & -1.29 & -1.32 & -1.77 & -1.12 & -1.53 & -1.15 & -1.55 & -1.04 & -1.41 \\
\hline Eritrea & & & -0.99 & -1.33 & -0.95 & -1.29 & -0.99 & -1.33 & -0.87 & -1.18 & -0.99 & -1.33 & -0.91 & -1.23 \\
\hline Ethiopia & -1.15 & & -0.99 & -1.33 & -0.95 & -1.29 & -1.25 & -1.71 & -0.63 & -0.89 & -1.12 & -1.52 & -0.79 & -1.09 \\
\hline Gambia, The & -1.07 & -0.18 & -0.99 & -1.33 & -0.95 & -1.29 & -1.32 & -1.77 & -1.12 & -1.53 & -1.15 & -1.55 & -1.04 & -1.41 \\
\hline Ghana & -1.09 & & -0.99 & -1.33 & -0.95 & -1.29 & -1.32 & -1.77 & -1.12 & -1.53 & -1.15 & -1.55 & -1.04 & -1.41 \\
\hline Guinea & -1.1 & & -0.99 & -1.33 & -0.95 & -1.29 & -1.32 & -1.77 & -1.12 & -1.53 & -1.15 & -1.55 & -1.04 & -1.41 \\
\hline Guinea Bissau & & & -0.99 & -1.33 & -0.95 & -1.29 & -1.32 & -1.77 & -1.12 & -1.53 & -1.15 & -1.55 & -1.04 & -1.41 \\
\hline Haiti & & -0.56 & -1.29 & -1.75 & -1.06 & -1.44 & -1.33 & -1.85 & -1.23 & -1.72 & -1.31 & -1.80 & -1.14 & -1.58 \\
\hline India & -1.74 & -0.14 & -1.04 & -1.41 & -0.83 & -1.13 & -1.28 & -1.76 & -0.21 & -0.28 & -1.16 & -1.59 & -0.52 & -0.71 \\
\hline Kenya & -1.14 & -0.77 & -0.99 & -1.33 & -0.95 & -1.29 & -0.99 & -1.33 & -0.87 & -1.18 & -0.99 & -1.33 & -0.91 & -1.23 \\
\hline Kyrgyzstan & -1.03 & & -1.24 & -1.68 & -0.90 & -1.22 & -1.43 & -1.98 & -0.64 & -0.86 & -1.34 & -1.83 & -0.77 & -1.04 \\
\hline Lao People's Dem.Rep & & & -0.97 & -1.33 & -0.83 & -1.15 & -1.04 & -1.41 & -0.55 & -0.75 & -1.00 & -1.37 & -0.69 & -0.95 \\
\hline Madagascar & -1.17 & -0.26 & -1.22 & -1.66 & -1.22 & -1.68 & -1.08 & -1.45 & -0.63 & -0.87 & -1.15 & -1.56 & -0.93 & -1.27 \\
\hline Malawi & -1.07 & -0.94 & -1.03 & -1.37 & -0.52 & -0.74 & -1.07 & -1.42 & -0.80 & -1.12 & -1.05 & -1.40 & -0.66 & -0.93 \\
\hline Mali & -1.08 & & -0.99 & -1.33 & -0.95 & -1.29 & -1.32 & -1.77 & -1.12 & -1.53 & -1.15 & -1.55 & -1.04 & -1.41 \\
\hline Mauritania & & -0.45 & -0.99 & -1.33 & -0.95 & -1.29 & -1.32 & -1.77 & -1.12 & -1.53 & -1.15 & -1.55 & -1.04 & -1.41 \\
\hline Myanmar & & -0.04 & -0.97 & -1.33 & -0.83 & -1.15 & -0.67 & -0.87 & -0.49 & -0.66 & -0.82 & -1.10 & -0.66 & -0.90 \\
\hline Mozambique & & & -1.13 & -1.53 & $\begin{array}{l}-0.00 \\
-0.70\end{array}$ & -0.95 & -1.12 & $\begin{array}{l}-0.01 \\
-1.58\end{array}$ & $\begin{array}{l}-0.41 \\
-0.41\end{array}$ & -0.60 & $\begin{array}{l}-0.02 \\
-1.13\end{array}$ & -1.55 & $\begin{array}{l}-0.56 \\
-0.56\end{array}$ & $\begin{array}{l}-0.78 \\
\end{array}$ \\
\hline Nepal & -1.13 & & -1.01 & -1.38 & -0.47 & -0.62 & -1.15 & -1.58 & -0.83 & -1.15 & -1.08 & -1.48 & -0.65 & -0.88 \\
\hline Niger & -1.09 & & -0.99 & -1.33 & -0.95 & -1.29 & -1.32 & -1.77 & -1.12 & -1.53 & -1.15 & -1.55 & -1.04 & -1.41 \\
\hline Nigeria & -1.32 & -0.34 & -0.99 & -1.33 & -0.95 & -1.29 & -0.91 & -1.23 & -0.83 & -1.15 & -0.95 & -1.28 & -0.89 & -1.22 \\
\hline Pakistan & & -0.52 & -1.01 & -1.38 & -0.47 & -0.62 & -1.04 & -1.40 & -0.80 & -1.07 & -1.03 & -1.39 & -0.63 & -0.84 \\
\hline Papua New Guinea & -1.15 & -0.27 & -1.39 & -1.89 & -0.56 & -0.75 & -1.30 & -1.79 & -0.68 & $\begin{array}{l}-0.93 \\
-0.93\end{array}$ & -1.35 & -1.84 & $\begin{array}{l}-0.03 \\
-0.62\end{array}$ & -0.84 \\
\hline Rwanda & -1.07 & -0.12 & -0.99 & -1.33 & -0.95 & -1.29 & -0.99 & -1.33 & -0.87 & -1.18 & -0.99 & -1.33 & -0.91 & -1.23 \\
\hline Senegal & -1.09 & & -0.99 & -1.33 & -0.95 & -1.29 & -1.28 & -1.72 & -1.00 & -1.32 & -1.13 & -1.53 & -0.97 & -1.30 \\
\hline Sierra Leone & & & -0.99 & -1.33 & -0.95 & -1.29 & $\begin{array}{l}-1.32 \\
-1.32\end{array}$ & -1.77 & $\begin{array}{l}-1.12 \\
\end{array}$ & -1.53 & -1.15 & -1.55 & -1.04 & -1.41 \\
\hline Tajikistan & & & -1.24 & -1.68 & -0.90 & -1.22 & -1.06 & -1.42 & -0.87 & -1.18 & -1.15 & -1.55 & -0.88 & -1.20 \\
\hline Tanzania & -1.31 & & -0.94 & -1.26 & -0.72 & -0.98 & -0.94 & $\begin{array}{l}-1.32 \\
-1.30\end{array}$ & -0.60 & -0.87 & -0.94 & -1.28 & -0.66 & -0.92 \\
\hline Togo & -1.09 & & -0.99 & -1.33 & -0.95 & -1.29 & -1.32 & -1.77 & -1.12 & -1.53 & -1.15 & -1.55 & -1.04 & -1.41 \\
\hline Uganda & -1.26 & & -0.85 & -1.13 & -0.62 & -0.83 & -0.78 & -1.05 & -0.49 & -0.68 & -0.82 & -1.09 & -0.56 & -0.76 \\
\hline Uzbekistan & & & -1.24 & -1.68 & -0.90 & -1.22 & -1.06 & -1.42 & -0.87 & -1.18 & -1.15 & -1.55 & -0.88 & -1.20 \\
\hline Viet nam & & & -1.64 & -2.22 & -1.31 & -1.80 & -1.62 & -2.21 & -1.39 & -1.90 & -1.63 & -2.22 & -1.35 & -1.85 \\
\hline Zambia & -1.11 & -0.51 & -1.08 & -1.47 & -0.77 & -1.04 & -1.11 & -1.53 & -0.87 & -1.22 & -1.10 & -1.50 & -0.82 & -1.13 \\
\hline Zimbabwe & -1.11 & & -0.96 & -1.31 & -0.41 & -0.56 & -1.15 & -1.57 & -0.37 & -0.51 & -1.06 & -1.44 & -0.39 & -0.54 \\
\hline mean & -1.15 & -0.40 & -1.05 & -1.42 & -0.85 & -1.16 & -1.18 & -1.60 & -0.87 & -1.19 & -1.11 & -1.51 & -0.86 & -1.17 \\
\hline median & -1.10 & -0.40 & -0.99 & -1.33 & -0.95 & -1.29 & -1.15 & -1.58 & -0.87 & -1.18 & -1.15 & -1.55 & -0.91 & -1.23 \\
\hline stdev & 0.16 & 0.24 & 0.14 & 0.20 & 0.21 & 0.29 & 0.21 & 0.30 & 0.28 & 0.38 & 0.15 & 0.21 & 0.22 & 0.30 \\
\hline
\end{tabular}


Table 1. Import Demand Elasticities, cont'd.

GTAP2001

GTAP2004

\begin{tabular}{|c|c|c|c|c|c|c|c|c|c|c|c|c|c|c|}
\hline & \multirow[b]{3}{*}{ Kee et al. (2008) } & \multirow[b]{3}{*}{ Senhadji (1997) } & \multicolumn{4}{|c|}{ GTAP2001 } & \multicolumn{4}{|c|}{ GTAP2004 } & \multicolumn{4}{|c|}{ Average 2001-2004 } \\
\hline & & & \multirow[b]{2}{*}{ SR } & \multirow[b]{2}{*}{ LR } & \multicolumn{2}{|c|}{$\begin{array}{c}\text { Adjusted to include GE } \\
\text { effects }\end{array}$} & \multirow[b]{2}{*}{ SR } & \multirow[b]{2}{*}{ LR } & \multicolumn{2}{|c|}{$\begin{array}{c}\text { Adjusted to include GE } \\
\text { effects }\end{array}$} & \multirow[b]{2}{*}{ SR } & \multirow[b]{2}{*}{ LR } & \multicolumn{2}{|c|}{$\begin{array}{c}\begin{array}{c}\text { Adjusted to include GE } \\
\text { effects }\end{array} \\
\end{array}$} \\
\hline & & & & & SR & LR & & & SR & LR & & & SR & LR \\
\hline \multicolumn{15}{|c|}{ Lower middle income } \\
\hline Albania & -1.14 & & -1.22 & -1.66 & -0.57 & -0.77 & -1.16 & -1.60 & -0.71 & -1.01 & -1.19 & -1.63 & -0.64 & -0.89 \\
\hline Algeria & -1.24 & -0.06 & -0.94 & -1.27 & -0.86 & -1.16 & -1.12 & -1.50 & -1.08 & -1.46 & -1.03 & -1.39 & -0.97 & -1.31 \\
\hline Armenia & -1.07 & & -1.24 & -1.68 & -0.90 & -1.22 & -1.17 & -1.65 & -0.76 & -1.08 & -1.20 & -1.67 & -0.83 & -1.15 \\
\hline Azerbaijan & -1.12 & & -1.24 & -1.68 & -0.90 & -1.22 & -1.57 & -2.28 & -1.56 & -2.27 & -1.41 & -1.98 & -1.23 & -1.74 \\
\hline Belarus & -1.1 & & -1.24 & -1.68 & -0.90 & -1.22 & -1.74 & -2.42 & 0.00 & 0.00 & -1.49 & -2.05 & -0.45 & -0.61 \\
\hline Bolivia & -1.15 & & -1.11 & -1.51 & -0.99 & -1.36 & -0.90 & -1.22 & -0.53 & -0.73 & -1.01 & -1.36 & -0.76 & -1.04 \\
\hline Bulgaria & -1.12 & & -1.56 & -2.12 & -0.95 & -1.31 & -1.66 & -2.27 & -1.11 & -1.54 & -1.61 & -2.20 & -1.03 & -1.42 \\
\hline Cameroon & -1.25 & -0.76 & -0.99 & -1.33 & -0.95 & -1.29 & -1.02 & -1.40 & -1.03 & -1.41 & -1.01 & -1.37 & -0.99 & -1.35 \\
\hline Cape Verde & -1.02 & & -0.99 & -1.33 & -0.95 & -1.29 & -1.32 & -1.77 & -1.12 & -1.53 & -1.15 & -1.55 & -1.04 & -1.41 \\
\hline China & -1.44 & -0.19 & -1.19 & -1.65 & -0.31 & -0.43 & -1.36 & -1.88 & -0.57 & -0.78 & -1.27 & -1.77 & -0.44 & -0.61 \\
\hline Colombia & -1.45 & -0.63 & -1.01 & -1.38 & -0.81 & -1.11 & -1.00 & -1.36 & -0.73 & -1.00 & -1.01 & -1.37 & -0.77 & -1.05 \\
\hline Congo, Republic of & -1.05 & -0.42 & -0.99 & -1.33 & -0.95 & -1.29 & -1.02 & -1.40 & -1.03 & -1.41 & -1.01 & -1.37 & -0.99 & -1.35 \\
\hline Dominican Republic & & -0.32 & -1.29 & -1.75 & -1.06 & -1.44 & -1.33 & -1.85 & -1.23 & -1.72 & -1.31 & -1.80 & -1.14 & -1.58 \\
\hline Ecuador & & & -1.11 & -1.51 & -0.99 & -1.36 & -1.20 & -1.65 & -1.13 & -1.56 & -1.16 & -1.58 & -1.06 & -1.46 \\
\hline Egypt & -1.31 & & -0.94 & -1.27 & -0.86 & -1.16 & -1.18 & -1.62 & -0.61 & -0.84 & -1.06 & -1.45 & -0.74 & -1.00 \\
\hline El Salvador & -1.2 & & -1.34 & -1.83 & -1.04 & -1.43 & -1.36 & -1.86 & -0.62 & -0.84 & -1.35 & -1.85 & -0.83 & -1.13 \\
\hline Georgia & -1.14 & & -1.24 & -1.68 & -0.90 & -1.22 & -1.19 & -1.63 & -0.63 & -0.86 & -1.22 & -1.66 & -0.76 & -1.04 \\
\hline Guatemala & -1.22 & & -1.34 & -1.83 & -1.04 & -1.43 & -1.06 & -1.46 & -0.66 & -0.92 & -1.20 & -1.65 & -0.85 & -1.17 \\
\hline Guyana & -1.05 & & -1.19 & -1.61 & -0.77 & -1.04 & -1.33 & -1.85 & -1.00 & -1.37 & -1.26 & -1.73 & -0.89 & -1.21 \\
\hline Honduras & -1.07 & -0.14 & -1.34 & -1.83 & -1.04 & -1.43 & -1.36 & -1.86 & -0.62 & -0.84 & -1.35 & -1.85 & -0.83 & -1.13 \\
\hline Indonesia & -1.38 & -0.62 & -1.08 & -1.47 & -0.84 & -1.15 & -0.97 & -1.32 & -0.62 & -0.86 & -1.02 & -1.40 & -0.73 & -1.00 \\
\hline Iran & -1.32 & & -0.99 & -1.34 & -0.81 & -1.10 & -1.21 & -1.64 & -1.18 & -1.60 & -1.10 & -1.49 & -0.99 & -1.35 \\
\hline Jordan & -1.08 & & -0.99 & -1.34 & -0.81 & -1.10 & -0.93 & -1.27 & -0.75 & -1.05 & -0.96 & -1.30 & -0.78 & -1.07 \\
\hline Kiribati & -1.01 & & -1.39 & -1.89 & -0.56 & -0.75 & -1.30 & -1.79 & -0.68 & -0.93 & -1.35 & -1.84 & -0.62 & -0.84 \\
\hline Lesotho & -1.02 & & -1.38 & -1.90 & -0.49 & -0.66 & -1.32 & -1.79 & -0.52 & -0.70 & -1.35 & -1.84 & -0.50 & -0.68 \\
\hline Macedonia & -1.12 & & -1.42 & -1.92 & -0.63 & -0.86 & -1.29 & -1.75 & -0.96 & -1.31 & -1.36 & -1.83 & -0.79 & -1.09 \\
\hline Maldives & -1.03 & & -1.01 & -1.38 & -0.47 & -0.62 & -1.15 & -1.58 & -0.83 & -1.15 & -1.08 & -1.48 & -0.65 & -0.88 \\
\hline Moldova & -1.07 & & -1.24 & -1.68 & -0.90 & -1.22 & -1.96 & -2.65 & -1.70 & -2.31 & -1.60 & -2.17 & -1.30 & -1.77 \\
\hline Mongolia & -1.03 & & -1.19 & -1.64 & -1.02 & -1.41 & -1.23 & -1.69 & -0.60 & -0.83 & -1.21 & -1.66 & -0.81 & -1.12 \\
\hline Morocco & -1.21 & -0.21 & -1.16 & -1.59 & -0.81 & -1.11 & -1.29 & -1.77 & -1.12 & -1.52 & -1.22 & -1.68 & -0.97 & -1.32 \\
\hline Namibia & -1.06 & & -1.38 & -1.90 & -0.49 & -0.66 & -1.32 & -1.79 & -0.52 & -0.70 & -1.35 & -1.84 & -0.50 & -0.68 \\
\hline Nicaragua & -1.06 & -0.15 & -1.34 & -1.83 & -1.04 & -1.43 & -1.18 & -1.60 & -0.91 & -1.23 & -1.26 & -1.72 & -0.97 & -1.33 \\
\hline Paraguay & -1.15 & -0.16 & -1.19 & -1.61 & -0.77 & -1.04 & -1.23 & -1.68 & -0.82 & -1.15 & -1.21 & -1.65 & -0.80 & -1.09 \\
\hline Peru & -1.5 & -0.32 & -0.98 & -1.33 & -0.77 & -1.05 & -0.90 & -1.24 & -0.63 & -0.87 & -0.94 & -1.29 & -0.70 & -0.96 \\
\hline Philippines & -1.15 & -0.36 & -1.77 & -2.40 & -0.52 & -0.73 & -1.63 & -2.24 & -0.53 & -0.72 & -1.70 & -2.32 & -0.53 & -0.72 \\
\hline Syrian Arab Republic & & & -0.99 & -1.34 & -0.81 & -1.10 & -0.93 & -1.27 & -0.75 & -1.05 & -0.96 & -1.30 & -0.78 & -1.07 \\
\hline Sri Lanka & -1.14 & & -1.30 & -1.77 & -0.70 & -0.95 & -1.29 & -1.72 & -0.69 & -0.90 & -1.30 & -1.75 & -0.69 & -0.92 \\
\hline Sudan & -1.39 & & -0.99 & -1.33 & -0.95 & -1.29 & -0.99 & -1.33 & -0.87 & -1.18 & -0.99 & -1.33 & -0.91 & -1.23 \\
\hline Swaziland & -1.05 & & -1.38 & -1.90 & -0.49 & -0.66 & -1.32 & -1.79 & -0.52 & -0.70 & -1.35 & -1.84 & -0.50 & -0.68 \\
\hline Thailand & -1.18 & -0.51 & -1.62 & -2.24 & -0.36 & -0.49 & -1.79 & -2.47 & -0.21 & -0.28 & -1.71 & -2.35 & -0.28 & -0.39 \\
\hline Tunisia & -1.11 & & -1.50 & -2.06 & -1.09 & -1.50 & -1.68 & -2.38 & -1.29 & -1.86 & -1.59 & -2.22 & -1.19 & -1.68 \\
\hline Turkmenistan & -1.04 & & -1.24 & -1.68 & -0.90 & -1.22 & -1.06 & -1.42 & -0.87 & -1.18 & -1.15 & -1.55 & -0.88 & -1.20 \\
\hline Ukraine & -1.19 & & -1.24 & -1.68 & -0.90 & -1.22 & -1.79 & -2.44 & -0.39 & -0.54 & -1.52 & -2.06 & -0.64 & -0.88 \\
\hline mean & -1.16 & -0.35 & -1.22 & -1.66 & -0.81 & -1.10 & -1.27 & -1.75 & -0.81 & -1.11 & -1.25 & -1.70 & -0.81 & -1.11 \\
\hline median & -1.13 & -0.32 & -1.24 & -1.68 & -0.86 & -1.16 & -1.23 & -1.69 & -0.75 & -1.05 & -1.22 & -1.67 & -0.80 & -1.09 \\
\hline stdev & 0.13 & 0.22 & 0.20 & 0.27 & 0.20 & 0.28 & 0.26 & 0.37 & 0.33 & 0.46 & 0.21 & 0.29 & 0.22 & 0.31 \\
\hline
\end{tabular}


Table 1. Import Demand Elasticities, cont'd

\begin{tabular}{|c|c|c|c|c|c|c|c|c|c|c|c|c|c|c|}
\hline & \multirow[b]{3}{*}{ Kee et al. (2008) } & \multirow[b]{3}{*}{ Senhadji (1997) } & \multicolumn{4}{|c|}{ GTAP2001 } & \multicolumn{4}{|c|}{ GTAP2004 } & \multicolumn{4}{|c|}{ Average 2001-2004 } \\
\hline & & & \multirow[b]{2}{*}{ SR } & \multirow[b]{2}{*}{ LR } & \multicolumn{2}{|c|}{$\begin{array}{c}\text { Adjusted to include GE } \\
\text { effects }\end{array}$} & \multirow[b]{2}{*}{ SR } & \multirow[b]{2}{*}{ LR } & \multicolumn{2}{|c|}{$\begin{array}{c}\text { Adjusted to include GE } \\
\text { effects }\end{array}$} & \multirow[b]{2}{*}{ SR } & \multirow[b]{2}{*}{ LR } & \multicolumn{2}{|c|}{$\begin{array}{c}\text { Adjusted to include GE } \\
\text { effects }\end{array}$} \\
\hline & & & & & SR & LR & & & SR & LR & & & SR & LR \\
\hline \multicolumn{15}{|c|}{ Upper middle income } \\
\hline Argentina & -1.86 & -0.64 & -1.20 & -1.67 & -1.00 & -1.41 & -1.24 & -1.68 & -1.05 & -1.43 & -1.22 & -1.67 & -1.03 & -1.42 \\
\hline Belize & -1.07 & & -1.34 & -1.83 & -1.04 & -1.43 & -1.36 & -1.86 & -0.62 & -0.84 & -1.35 & -1.85 & -0.83 & -1.13 \\
\hline Botswana & -1.04 & & -1.18 & -1.62 & -0.59 & -0.80 & -1.13 & -1.56 & -1.09 & -1.50 & -1.16 & -1.59 & -0.84 & -1.15 \\
\hline Brazil & -2.17 & -0.3 & -1.20 & -1.66 & -0.87 & -1.20 & -1.04 & -1.41 & -0.65 & -0.86 & -1.12 & -1.53 & -0.76 & -1.03 \\
\hline Chile & -1.27 & -0.09 & -1.26 & -1.74 & -0.98 & -1.37 & -1.29 & -1.73 & -1.08 & -1.43 & -1.28 & -1.74 & -1.03 & -1.40 \\
\hline Costa Rica & -1.1 & -0.55 & -1.34 & -1.83 & -1.04 & -1.43 & -1.36 & -1.84 & -0.43 & -0.61 & -1.35 & -1.84 & -0.73 & -1.02 \\
\hline Croatia & -1.19 & & -1.86 & -2.53 & -0.66 & -0.87 & -2.06 & -2.89 & -0.87 & -1.21 & -1.96 & -2.71 & -0.76 & -1.04 \\
\hline Dominica & -1.06 & & -1.29 & -1.75 & -1.06 & -1.44 & -1.33 & -1.85 & -1.23 & -1.72 & -1.31 & -1.80 & -1.14 & -1.58 \\
\hline Gabon & -1.16 & -0.27 & -0.99 & -1.33 & -0.95 & -1.29 & -1.02 & -1.40 & -1.03 & -1.41 & -1.01 & -1.37 & -0.99 & -1.35 \\
\hline Grenada & -1.03 & & -1.29 & -1.75 & -1.06 & -1.44 & -1.33 & -1.85 & -1.23 & -1.72 & -1.31 & -1.80 & -1.14 & -1.58 \\
\hline Jamaica & -1.14 & & -1.29 & -1.75 & -1.06 & -1.44 & -1.33 & -1.85 & -1.23 & -1.72 & -1.31 & -1.80 & -1.14 & -1.58 \\
\hline Kazakhstan & -1.12 & & -1.24 & -1.68 & -0.90 & -1.22 & -1.20 & -1.65 & -0.84 & -1.18 & -1.22 & -1.66 & -0.87 & -1.20 \\
\hline Latvia & -1.11 & & -1.49 & -2.03 & -1.08 & -1.48 & -1.43 & -1.96 & -1.10 & -1.51 & -1.46 & -2.00 & -1.09 & -1.49 \\
\hline Lebanon & -1.13 & & -0.99 & -1.34 & -0.81 & -1.10 & -0.93 & -1.27 & -0.75 & -1.05 & -0.96 & -1.30 & -0.78 & -1.07 \\
\hline Libya & & & -0.94 & -1.27 & -0.86 & -1.16 & -1.12 & -1.50 & -1.08 & -1.46 & -1.03 & -1.39 & -0.97 & -1.31 \\
\hline Lithuania & -1.17 & & -1.62 & -2.24 & -0.79 & -1.11 & -1.74 & -2.40 & -0.76 & -1.03 & -1.68 & -2.32 & -0.77 & -1.07 \\
\hline Malaysia & -1.08 & & -1.60 & -2.19 & -0.52 & -0.73 & -1.49 & -2.06 & -0.51 & -0.71 & -1.54 & -2.12 & -0.51 & -0.72 \\
\hline Mauritius & -1.08 & -0.26 & -0.93 & -1.25 & -0.60 & -0.83 & -1.36 & -1.84 & -0.29 & -0.40 & -1.15 & -1.54 & -0.45 & -0.61 \\
\hline Mexico & -1.34 & -0.37 & -1.13 & -1.56 & -0.57 & -0.80 & -1.47 & -2.05 & -0.19 & -0.28 & -1.30 & -1.81 & -0.38 & -0.54 \\
\hline Panama & -1.19 & -0.01 & -1.34 & -1.83 & -1.04 & -1.43 & -1.15 & -1.59 & -0.70 & -0.99 & -1.25 & -1.71 & -0.87 & -1.21 \\
\hline Poland & -1.32 & & -1.26 & -1.71 & -1.02 & -1.37 & -1.34 & -1.82 & -0.90 & -1.21 & -1.30 & -1.77 & -0.96 & -1.29 \\
\hline Romania & -1.19 & & -1.18 & -1.60 & -0.83 & -1.12 & -1.27 & -1.73 & -0.51 & -0.70 & -1.22 & -1.67 & -0.67 & -0.91 \\
\hline Russian Federation & -1.57 & & -0.96 & -1.30 & -0.84 & -1.14 & -0.97 & -1.32 & -0.84 & -1.15 & -0.96 & -1.31 & -0.84 & -1.15 \\
\hline Seychelles & -1.06 & & -0.93 & -1.25 & -0.60 & -0.83 & -0.99 & -1.33 & -0.87 & -1.18 & -0.96 & -1.29 & -0.73 & -1.00 \\
\hline South Africa & -1.43 & -0.53 & -1.17 & -1.62 & -0.64 & -0.87 & -1.32 & -1.83 & -0.71 & -0.99 & -1.25 & -1.72 & -0.68 & -0.93 \\
\hline St. Kitts and Nevis & -1.02 & & -1.29 & -1.75 & -1.06 & -1.44 & -1.33 & -1.85 & -1.23 & -1.72 & -1.31 & -1.80 & -1.14 & -1.58 \\
\hline St. Lucia & -1.07 & & -1.29 & -1.75 & -1.06 & -1.44 & -1.33 & -1.85 & -1.23 & -1.72 & -1.31 & -1.80 & -1.14 & -1.58 \\
\hline St. Vincent \& Grens. & -1.02 & & -1.29 & -1.75 & -1.06 & -1.44 & -1.33 & -1.85 & -1.23 & -1.72 & -1.31 & -1.80 & -1.14 & -1.58 \\
\hline Suriname & -1.04 & & -1.19 & -1.61 & -0.77 & -1.04 & -1.33 & -1.85 & -1.00 & -1.37 & -1.26 & -1.73 & -0.89 & -1.21 \\
\hline Turkey & -1.32 & 0.03 & -1.25 & -1.70 & -0.70 & -0.95 & -1.30 & -1.80 & -0.91 & -1.26 & -1.28 & -1.75 & -0.80 & -1.11 \\
\hline Uruguay & -1.44 & -0.2 & -1.23 & -1.69 & -0.89 & -1.24 & -1.54 & -2.08 & -0.69 & -0.94 & -1.38 & -1.89 & -0.79 & -1.09 \\
\hline Venezuela & -1.48 & & -1.34 & -1.87 & -1.29 & -1.81 & -0.99 & -1.31 & -0.88 & $\begin{array}{l}-0.94 \\
-1.19\end{array}$ & $\begin{array}{l}-1.50 \\
-1.16\end{array}$ & $\begin{array}{l}-1.09 \\
-1.59\end{array}$ & -1.09 & $\begin{array}{l}-1.09 \\
-1.50\end{array}$ \\
\hline Mean & -1.23 & -0.29 & -1.25 & -1.70 & -0.88 & -1.21 & -1.29 & -1.78 & -0.87 & -1.19 & -1.27 & -1.74 & -0.87 & -1.20 \\
\hline Median & -1.14 & -0.27 & -1.25 & -1.71 & -0.89 & -1.23 & -1.33 & -1.83 & -0.87 & -1.20 & -1.28 & -1.74 & -0.85 & -1.18 \\
\hline Stdev & 0.26 & 0.22 & 0.20 & 0.28 & 0.19 & 0.27 & 0.23 & 0.33 & 0.28 & 0.40 & 0.20 & 0.29 & 0.21 & 0.29 \\
\hline
\end{tabular}


Table 1. Import Demand Elasticities cont'd

\begin{tabular}{|c|c|c|c|c|c|c|c|c|c|c|c|c|c|c|}
\hline & \multirow[b]{3}{*}{ Kee et al. (2008) } & \multirow[b]{3}{*}{ Senhadji (1997) } & \multicolumn{4}{|c|}{ GTAP2001 } & \multicolumn{4}{|c|}{ GTAP2004 } & \multicolumn{4}{|c|}{ Average 2001-2004 } \\
\hline & & & \multirow[b]{2}{*}{ SR } & \multirow[b]{2}{*}{ LR } & \multicolumn{2}{|c|}{$\begin{array}{c}\text { Adjusted to include GE } \\
\text { effects }\end{array}$} & \multirow[b]{2}{*}{ SR } & \multirow[b]{2}{*}{ LR } & \multicolumn{2}{|c|}{$\begin{array}{c}\text { Adjusted to include GE } \\
\text { effects }\end{array}$} & \multirow[b]{2}{*}{ SR } & \multirow[b]{2}{*}{ LR } & \multicolumn{2}{|c|}{$\begin{array}{c}\text { Adjusted to include GE } \\
\text { effects }\end{array}$} \\
\hline & & & & & SR & LR & & & SR & LR & & & SR & LR \\
\hline \multicolumn{15}{|c|}{ High income: nonOECD } \\
\hline Antigua and Barbuda & -1.08 & & -1.29 & -1.75 & -1.06 & -1.44 & -1.33 & -1.85 & -1.23 & -1.72 & -1.31 & -1.80 & -1.14 & -1.58 \\
\hline Bahamas, The & -1.09 & & -1.29 & -1.75 & -1.06 & -1.44 & -1.33 & -1.85 & -1.23 & -1.72 & -1.31 & -1.80 & -1.14 & -1.58 \\
\hline Bahrain & -1.09 & & -0.99 & -1.34 & -0.81 & -1.10 & -0.93 & -1.27 & -0.75 & -1.05 & -0.96 & -1.30 & -0.78 & -1.07 \\
\hline Barbados & -1.12 & & -1.29 & -1.75 & -1.06 & -1.44 & -1.33 & -1.85 & -1.23 & -1.72 & -1.31 & -1.80 & -1.14 & -1.58 \\
\hline Bermuda & -1.06 & & -1.99 & -2.66 & -1.60 & -2.12 & -1.92 & -2.59 & -1.71 & -2.31 & -1.95 & -2.62 & -1.66 & -2.21 \\
\hline Brunei & -1.08 & & -0.97 & -1.33 & -0.83 & -1.15 & -0.94 & -1.24 & -0.73 & -0.98 & -0.95 & -1.28 & -0.78 & -1.07 \\
\hline Cyprus & -1.13 & & -2.63 & -3.68 & -2.60 & -3.65 & -1.49 & -2.02 & -1.04 & -1.37 & -2.06 & -2.85 & -1.82 & -2.51 \\
\hline Estonia & -1.05 & & -1.61 & -2.20 & -0.49 & -0.67 & -2.40 & -3.34 & -1.77 & -2.51 & -2.00 & -2.77 & -1.13 & -1.59 \\
\hline French Polynesia & -1.06 & & -1.39 & -1.89 & -0.56 & -0.75 & -1.30 & -1.79 & -0.68 & -0.93 & -1.35 & -1.84 & -0.62 & -0.84 \\
\hline Greenland & -1.04 & & -1.99 & -2.66 & -1.60 & -2.12 & -1.92 & -2.59 & -1.71 & -2.31 & -1.95 & -2.62 & -1.66 & -2.21 \\
\hline Hong Kong & -1.05 & & -1.48 & -2.03 & -0.91 & -1.24 & -1.47 & -2.04 & -0.66 & -0.92 & -1.48 & -2.03 & -0.79 & -1.08 \\
\hline Israel & -1.2 & -0.1 & -0.99 & -1.34 & -0.81 & -1.10 & -0.93 & -1.27 & -0.75 & -1.05 & -0.96 & -1.30 & -0.78 & -1.07 \\
\hline Macau & -1.11 & & -1.19 & -1.64 & -1.02 & -1.41 & -1.23 & -1.69 & -0.60 & -0.83 & -1.21 & -1.66 & -0.81 & -1.12 \\
\hline Malta & -1.11 & & -1.77 & -2.41 & -0.90 & -1.25 & -1.58 & -2.19 & -0.72 & -1.03 & -1.67 & -2.30 & -0.81 & -1.14 \\
\hline N. Caledonia & -1.07 & & -1.39 & -1.89 & -0.56 & -0.75 & -1.30 & -1.79 & -0.68 & -0.93 & -1.35 & -1.84 & -0.62 & -0.84 \\
\hline Oman & -1.11 & & -0.99 & -1.34 & -0.81 & -1.10 & -0.93 & -1.27 & -0.75 & -1.05 & -0.96 & -1.30 & -0.78 & -1.07 \\
\hline Saudi Arabia & -1.3 & & -0.99 & -1.34 & -0.81 & -1.10 & -0.93 & -1.27 & -0.75 & -1.05 & -0.96 & -1.30 & -0.78 & -1.07 \\
\hline Singapore & -1.05 & & -2.91 & -3.99 & -0.64 & -0.90 & -2.55 & -3.53 & -0.39 & -0.54 & -2.73 & -3.76 & -0.51 & -0.72 \\
\hline Slovenia & -1.1 & & -1.60 & -2.19 & -0.63 & -0.82 & -1.55 & -2.14 & -0.70 & -0.92 & -1.57 & -2.16 & -0.66 & -0.87 \\
\hline Taiwan & -1.17 & & -2.15 & -3.00 & -0.25 & -0.31 & -2.62 & -3.64 & 0.26 & 0.38 & -2.39 & -3.32 & 0.01 & 0.03 \\
\hline Trinidad and Tobago & -1.15 & -0.49 & -1.29 & -1.75 & -1.06 & -1.44 & -1.33 & -1.85 & -1.23 & -1.72 & -1.31 & -1.80 & -1.14 & -1.58 \\
\hline Mean & -1.11 & -0.30 & -1.53 & -2.09 & -0.95 & -1.30 & -1.49 & -2.05 & -0.91 & -1.25 & -1.51 & -2.07 & -0.93 & -1.28 \\
\hline Median & -1.09 & -0.30 & -1.39 & -1.89 & -0.83 & -1.15 & -1.33 & -1.85 & -0.75 & -1.05 & -1.35 & -1.84 & -0.79 & -1.08 \\
\hline Stdev & 0.06 & 0.28 & 0.54 & 0.75 & 0.50 & 0.69 & 0.52 & 0.73 & 0.48 & 0.66 & 0.50 & 0.70 & 0.42 & 0.58 \\
\hline
\end{tabular}


Table 1. Import Demand Elasticities, concluded

\begin{tabular}{|c|c|c|c|c|c|c|c|c|c|c|c|c|c|c|}
\hline & \multirow[b]{3}{*}{ Kee et al. (2008) } & \multirow[b]{3}{*}{ Senhadji (1997) } & \multicolumn{8}{|c|}{ GTAP2001 } & \multicolumn{4}{|c|}{ Average 2001-2004 } \\
\hline & & & \multirow[b]{2}{*}{ SR } & \multirow[b]{2}{*}{ LR } & \multicolumn{2}{|c|}{$\begin{array}{l}\text { Adjusted to include GE } \\
\text { effects }\end{array}$} & \multirow[b]{2}{*}{ SR } & \multirow[b]{2}{*}{ LR } & \multicolumn{2}{|c|}{$\begin{array}{l}\text { Adjusted to include GE } \\
\text { effects }\end{array}$} & \multirow[b]{2}{*}{ SR } & \multirow[b]{2}{*}{ LR } & \multicolumn{2}{|c|}{$\begin{array}{l}\text { Adjusted to include GE } \\
\text { effects }\end{array}$} \\
\hline & & & & & SR & LR & & & SR & LR & & & SR & LR \\
\hline \multicolumn{15}{|l|}{$\begin{array}{l}\text { High income: OECD } \\
\end{array}$} \\
\hline Australia & -1.46 & -0.34 & -1.26 & -1.76 & -1.15 & -1.61 & -1.24 & -1.73 & -1.15 & -1.61 & -1.25 & -1.74 & -1.15 & -1.61 \\
\hline Austria & -1.25 & -0.33 & -1.46 & -1.97 & -0.68 & -0.89 & -1.52 & -2.08 & -0.65 & -0.86 & -1.49 & -2.02 & -0.67 & -0.88 \\
\hline Belgium & -1.14 & -0.17 & -2.88 & -3.88 & 0.12 & 0.14 & -2.36 & -3.29 & -0.95 & -1.26 & -2.62 & -3.58 & -0.41 & -0.56 \\
\hline Canada & -1.28 & -0.57 & -1.42 & -1.93 & $\begin{array}{l}-0.70 \\
-0.70\end{array}$ & -0.94 & -1.27 & $\begin{array}{l}-1.72 \\
\end{array}$ & -0.51 & -0.72 & -1.34 & -1.82 & $\begin{array}{l}-0.71 \\
-0.61\end{array}$ & -0.83 \\
\hline Czech Republic & -1.15 & & -1.64 & -2.25 & -0.95 & -1.29 & -1.82 & -2.48 & -0.84 & -1.12 & -1.73 & -2.37 & -0.90 & -1.20 \\
\hline Denmark & -1.38 & -0.14 & -1.55 & -2.13 & -0.40 & -0.54 & -1.36 & -1.89 & -0.70 & -1.02 & -1.46 & -2.01 & -0.55 & -0.78 \\
\hline Finland & -1.37 & & -1.57 & -2.21 & -0.08 & -0.07 & -1.50 & -2.10 & 0.04 & 0.05 & -1.54 & -2.16 & -0.02 & -0.01 \\
\hline France & -1.47 & -0.22 & -1.23 & -1.68 & -0.73 & -1.04 & -1.44 & -1.97 & -0.73 & -1.02 & -1.34 & -1.83 & -0.73 & -1.03 \\
\hline Germany & -1.43 & -0.06 & -1.43 & -1.97 & -0.05 & -0.02 & -1.48 & -2.05 & -0.16 & -0.17 & -1.46 & -2.01 & -0.10 & -0.10 \\
\hline Greece & -1.37 & -0.4 & -1.24 & -1.67 & -0.35 & -0.48 & -1.31 & -1.83 & -1.24 & -1.75 & -1.27 & -1.75 & -0.80 & -1.11 \\
\hline Hungary & -1.11 & & -1.58 & -2.16 & -0.67 & -0.90 & -1.74 & -2.37 & -0.57 & -0.76 & -1.66 & -2.27 & -0.62 & -0.83 \\
\hline Iceland & -1.2 & -0.44 & -1.44 & -1.96 & -1.21 & -1.66 & -1.42 & -1.94 & -0.93 & -1.26 & -1.43 & -1.95 & -1.07 & -1.46 \\
\hline Ireland & -1.2 & & -1.67 & -2.26 & -0.04 & -0.06 & -1.66 & -2.25 & -0.43 & -0.61 & -1.67 & -2.26 & -0.24 & -0.34 \\
\hline Italy & -1.35 & -0.21 & -1.48 & -2.04 & -0.85 & -1.17 & -1.43 & -1.97 & -0.56 & -0.76 & -1.46 & -2.01 & -0.70 & -0.97 \\
\hline Japan & -1.83 & -0.14 & -1.20 & -1.67 & -0.54 & -0.74 & -1.27 & -1.78 & -0.56 & -0.77 & -1.24 & -1.72 & -0.55 & -0.75 \\
\hline Korea & -1.24 & -0.17 & -2.00 & -2.80 & -0.19 & -0.22 & -2.01 & -2.81 & -0.16 & -0.20 & -2.00 & -2.80 & -0.17 & -0.21 \\
\hline Luxembourg & -1.05 & & -1.97 & -2.67 & -0.74 & -1.03 & -3.28 & -4.54 & -3.06 & -4.24 & -2.62 & -3.61 & -1.90 & -2.63 \\
\hline Netherlands & -1.15 & & -1.68 & -2.29 & $\begin{array}{l}-0.14 \\
-0.52\end{array}$ & -0.69 & $\begin{array}{l}-3.59 \\
-1.59\end{array}$ & -2.15 & -0.56 & -0.76 & -1.64 & -2.22 & -0.54 & -0.73 \\
\hline New Zealand & -1.27 & -0.38 & -1.17 & -1.58 & -0.75 & -1.00 & -1.20 & -1.63 & -0.66 & -0.88 & -1.19 & -1.60 & -0.70 & -0.94 \\
\hline Norway & -1.41 & -0.41 & -1.44 & -1.96 & -1.21 & -1.66 & -1.24 & -1.69 & -1.12 & -1.55 & -1.34 & -1.83 & -1.17 & -1.61 \\
\hline Portugal & -1.25 & -0.38 & -1.65 & -2.26 & -1.11 & -1.52 & -1.54 & -2.10 & $\begin{array}{l}-1.03 \\
\end{array}$ & -1.41 & -1.59 & -2.18 & -1.07 & -1.46 \\
\hline Slovakia & $\begin{array}{l}-1.09 \\
-1.09\end{array}$ & & -1.75 & -2.43 & -0.64 & -0.87 & $\begin{array}{r}-1.64 \\
-1.69\end{array}$ & $\begin{array}{l}-2.34 \\
-2.34\end{array}$ & $\begin{array}{l}-0.57 \\
-0.57\end{array}$ & $\begin{array}{l}-1.41 \\
-0.78\end{array}$ & $\begin{array}{l}-1.72 \\
-1.72\end{array}$ & $\begin{array}{l}-2.10 \\
-2.39\end{array}$ & -0.61 & $\begin{array}{l}-0.83 \\
-0.83\end{array}$ \\
\hline Spain & -1.33 & -0.35 & -1.37 & -1.89 & -1.04 & -1.43 & -1.24 & -1.69 & -0.90 & -1.22 & -1.31 & -1.79 & -0.97 & -1.33 \\
\hline Sweden & -1.37 & -0.06 & -1.70 & -2.36 & 0.11 & 0.19 & -1.60 & -2.23 & -0.09 & -0.10 & -1.65 & -2.29 & 0.01 & 0.04 \\
\hline Switzerland & -1.32 & -0.22 & -1.40 & -1.92 & -0.83 & -1.17 & -1.46 & -1.99 & -0.28 & -0.38 & -1.43 & -1.96 & -0.56 & -0.78 \\
\hline United States of America & $\begin{array}{l}-1.02 \\
-2.09\end{array}$ & $\begin{array}{l}-0.22 \\
-0.25\end{array}$ & -1.14 & -1.59 & $\begin{array}{l}-1.07 \\
-1.07 \quad x-1\end{array}$ & -1.50 & $\begin{array}{r}-1.40 \\
-1.29\end{array}$ & $\begin{array}{r}-1.79 \\
-1.79\end{array}$ & -1.11 & -1.55 & $\begin{array}{r}-1.40 \\
-1.21\end{array}$ & -1.69 & -1.09 & -1.52 \\
\hline United Kingdom & -1.42 & -0.01 & -1.41 & -1.95 & -1.23 & -1.72 & -1.32 & -1.82 & -1.17 & -1.63 & -1.37 & -1.89 & -1.20 & -1.68 \\
\hline Mean & -1.33 & -0.26 & -1.55 & -2.12 & -0.65 & -0.89 & -1.57 & -2.16 & -0.77 & -1.05 & -1.56 & -2.14 & -0.71 & -0.97 \\
\hline Median & -1.32 & -0.24 & -1.46 & -1.97 & -0.70 & -0.94 & -1.46 & -1.99 & -0.66 & -0.88 & -1.46 & -2.01 & -0.67 & -0.88 \\
\hline Stdev & 0.22 & 0.15 & 0.35 & 0.47 & 0.42 & 0.59 & 0.43 & 0.60 & 0.58 & 0.81 & 0.36 & 0.50 & 0.42 & 0.59 \\
\hline \multicolumn{15}{|l|}{ Overall } \\
\hline Mean & -1.20 & -0.32 & -1.27 & -1.73 & -0.83 & -1.13 & -1.33 & -1.82 & -0.84 & -1.16 & -1.30 & -1.78 & -0.83 & -1.14 \\
\hline Median & -1.13 & -0.30 & -1.24 & -1.68 & -0.89 & -1.22 & -1.31 & -1.77 & -0.84 & -1.15 & -1.25 & -1.72 & -0.82 & -1.13 \\
\hline Stdev & 0.19 & 0.21 & 0.33 & 0.46 & 0.31 & 0.43 & 0.35 & 0.49 & 0.38 & 0.53 & 0.32 & 0.45 & 0.30 & 0.41 \\
\hline
\end{tabular}

Notel: Income group: Economies are divided according to 2007 GNI per capita, calculated using the World Bank

Atlas method. The groups are: low income, \$935 or less; lower middle income, \$936 - \$3,705; upper middle income.

Note: The CGER import-demand elasticity (not shown) is calculated keeping import elasticities to -0.92 for all countries except China ( Im: -0.67), Malaysia (Im: - I) and Colombia (Im: - -1) 
Table 2: Export supply elasticities. This table reports export supply elasticities estimated in the literature, as well as export supply elasticities estimated using the method described in this paper. The format of this table follows the format of the table for import demand elasticities (see above).

Regarding the "general equilibrium" elasticities, it is possible that the import demand elasticity could be positive, while the export supply elasticity could be negative. Consider the import demand elasticities. If only the price of imported inputs changes, then the quantity demanded must fall, leading to a negative elasticity. When the price of exports is also allowed to change, then the net quantity demanded of imported inputs could actually increase. This is because a devaluation raises the domestic price of exports and leads to an increase export volume. Since the export sector uses imported inputs, this will lead to an increase in demand for imported inputs. The net impact on import volume depends on the impact of the rise in the price of imports (holding export volume constant) plus the impact through the expansion in exports. It turns out that the import demand elasticity is positive for one country, Sweden, as shown in table 1.

Similarly, the "general equilibrium" export supply elasticity could be negative. An increase in the price of exports alone will increase export volume; thus the "partial equilibrium" export supply elasticity is positive. When the price of imported inputs also changes, this will reduce export volume, since exports require imported inputs. Depending on the intensity with which exports use imported inputs, export volume could rise or fall on net. As shown in table 2, all export supply elasticities are positive. 
Table 2. Export Supply Elasticities

GTAP2004

GTAP2004
Adjusted to include GE

Average 2001-2004

\begin{tabular}{|c|c|c|c|c|c|c|c|c|c|c|c|c|c|}
\hline \multirow{3}{*}{\multicolumn{2}{|c|}{$\begin{array}{l}\text { Broda, Limao \& } \\
\text { Weinstein (2008) }\end{array}$}} & \multicolumn{4}{|c|}{ GTAP2001 } & \multicolumn{4}{|c|}{ GTAP2004 } & \multicolumn{4}{|c|}{$2001-2004$} \\
\hline & & \multirow[b]{2}{*}{ SR } & \multirow[b]{2}{*}{ LR } & \multicolumn{2}{|c|}{ Adjusted to include GE effects } & \multirow[b]{2}{*}{ SR } & \multirow[b]{2}{*}{ LR } & \multicolumn{2}{|c|}{$\begin{array}{c}\text { Adjusted to include GE } \\
\text { effects }\end{array}$} & \multirow[b]{2}{*}{ SR } & \multirow[b]{2}{*}{ LR } & \multicolumn{2}{|c|}{$\begin{array}{c}\text { Adjusted to include GE } \\
\text { effects }\end{array}$} \\
\hline & & & & SR & LR & & & SR & LR & & & SR & LR \\
\hline \multicolumn{14}{|l|}{ Low income } \\
\hline Bangladesh & & 2.20 & 3.04 & 1.40 & 1.92 & 2.09 & 2.91 & 1.31 & 1.83 & 2.15 & 2.97 & 1.35 & 1.87 \\
\hline Benin & & 0.30 & 0.36 & 0.28 & 0.33 & 0.47 & 0.60 & 0.31 & 0.40 & 0.39 & 0.48 & 0.29 & 0.36 \\
\hline Burkina Faso & & 0.30 & 0.36 & 0.28 & 0.33 & 0.47 & 0.60 & 0.31 & 0.40 & 0.39 & 0.48 & 0.29 & 0.36 \\
\hline Burundi & & 0.30 & 0.36 & 0.28 & 0.33 & 0.66 & 0.88 & 0.60 & 0.80 & 0.48 & 0.62 & 0.44 & 0.57 \\
\hline Central African Rep. & & 0.30 & 0.36 & 0.28 & 0.33 & 0.15 & 0.17 & 0.15 & 0.17 & 0.23 & 0.26 & 0.21 & 0.25 \\
\hline Chad & & 0.30 & 0.36 & 0.28 & 0.33 & 0.15 & 0.17 & 0.15 & 0.17 & 0.23 & 0.26 & 0.21 & 0.25 \\
\hline Cambodia & & 0.33 & 0.44 & 0.27 & 0.36 & 1.11 & 1.51 & 0.49 & 0.69 & 0.72 & 0.98 & 0.38 & 0.52 \\
\hline Comoros & & 0.30 & 0.36 & 0.28 & 0.33 & 0.66 & 0.88 & 0.60 & 0.80 & 0.48 & 0.62 & 0.44 & 0.57 \\
\hline Congo, Dem. Rep. & & 0.42 & 0.54 & 0.25 & 0.32 & 0.08 & 0.09 & 0.04 & 0.04 & 0.25 & 0.31 & 0.14 & 0.18 \\
\hline Côte d'Ivoire & & 0.30 & 0.36 & 0.28 & 0.33 & 0.47 & 0.60 & 0.31 & 0.40 & 0.39 & 0.48 & 0.29 & 0.36 \\
\hline Eritrea & & 0.30 & 0.36 & 0.28 & 0.33 & 0.66 & 0.88 & 0.60 & 0.80 & 0.48 & 0.62 & 0.44 & 0.57 \\
\hline Ethiopia & & 0.30 & 0.36 & 0.28 & 0.33 & 0.79 & 1.04 & 0.50 & 0.67 & 0.55 & 0.70 & 0.39 & 0.50 \\
\hline Gambia, The & & 0.30 & 0.36 & 0.28 & 0.33 & 0.47 & 0.60 & 0.31 & 0.40 & 0.39 & 0.48 & 0.29 & 0.36 \\
\hline Ghana & & 0.30 & 0.36 & 0.28 & 0.33 & 0.47 & 0.60 & 0.31 & 0.40 & 0.39 & 0.48 & 0.29 & 0.36 \\
\hline Guinea Bissau & & 0.30 & 0.36 & 0.28 & 0.33 & 0.47 & 0.60 & 0.31 & 0.40 & 0.39 & 0.48 & 0.29 & 0.36 \\
\hline Guinea & & 0.30 & 0.36 & 0.28 & 0.33 & 0.47 & 0.60 & 0.31 & 0.40 & 0.39 & 0.48 & 0.29 & 0.36 \\
\hline Haiti & & 0.98 & 1.31 & 0.80 & 1.07 & 0.62 & 0.87 & 0.55 & 0.77 & 0.80 & 1.09 & 0.68 & 0.92 \\
\hline India & & 0.81 & 1.11 & 0.65 & 0.88 & 1.61 & 2.22 & 0.81 & 1.12 & 1.21 & 1.66 & 0.73 & 1.00 \\
\hline Kenya & & 0.30 & 0.36 & 0.28 & 0.33 & 0.66 & 0.88 & 0.60 & 0.80 & 0.48 & 0.62 & 0.44 & 0.57 \\
\hline Kyrgyzstan & & 1.27 & 1.71 & 0.88 & 1.19 & 0.46 & 0.64 & 0.16 & 0.21 & 0.87 & 1.17 & 0.52 & 0.70 \\
\hline Lao People's Dem.Rep & & 0.33 & 0.44 & 0.27 & 0.36 & 0.84 & 1.12 & 0.50 & 0.67 & 0.59 & 0.78 & 0.39 & 0.52 \\
\hline Madagascar & & 0.74 & 0.91 & 0.75 & 0.93 & 0.86 & 1.14 & 0.59 & 0.79 & 0.80 & 1.03 & 0.67 & 0.86 \\
\hline Malawi & & 0.97 & 1.21 & 0.67 & 0.84 & 0.55 & 0.64 & 0.29 & 0.34 & 0.76 & 0.93 & 0.48 & 0.59 \\
\hline Mali & & 0.30 & 0.36 & 0.28 & 0.33 & 0.47 & 0.60 & 0.31 & 0.40 & 0.39 & 0.48 & 0.29 & 0.36 \\
\hline Mozambique & & 1.23 & 1.67 & 0.93 & 1.25 & 1.12 & 1.55 & 0.72 & 1.01 & 1.17 & 1.61 & 0.83 & 1.13 \\
\hline Mauritania & & 0.30 & 0.36 & 0.28 & 0.33 & 0.47 & 0.60 & 0.31 & 0.40 & 0.39 & 0.48 & 0.29 & 0.36 \\
\hline Myanmar & & 0.33 & 0.44 & 0.27 & 0.36 & 0.29 & 0.35 & 0.18 & 0.22 & 0.31 & 0.39 & 0.23 & 0.29 \\
\hline Nepal & & 1.31 & 1.82 & 0.92 & 1.28 & 1.00 & 1.37 & 0.79 & 1.08 & 1.16 & 1.59 & 0.86 & 1.18 \\
\hline Niger & & 0.30 & 0.36 & 0.28 & 0.33 & 0.47 & 0.60 & 0.31 & 0.40 & 0.39 & 0.48 & 0.29 & 0.36 \\
\hline Nigeria & & 0.30 & 0.36 & 0.28 & 0.33 & 0.02 & 0.02 & 0.00 & 0.00 & 0.16 & 0.19 & 0.14 & 0.17 \\
\hline Pakistan & & 1.31 & 1.82 & 0.92 & 1.28 & 0.69 & 0.95 & 0.35 & 0.48 & 1.00 & 1.38 & 0.64 & 0.88 \\
\hline Papua New Guinea & & 1.45 & 1.99 & 0.82 & 1.12 & 0.84 & 1.15 & 0.51 & 0.71 & 1.15 & 1.57 & 0.67 & 0.91 \\
\hline Rwanda & & 0.30 & 0.36 & 0.28 & 0.33 & 0.66 & 0.88 & 0.60 & 0.80 & 0.48 & 0.62 & 0.44 & 0.57 \\
\hline Senegal & & 0.30 & 0.36 & 0.28 & 0.33 & 0.80 & 1.14 & 0.44 & 0.62 & 0.55 & 0.75 & 0.36 & 0.47 \\
\hline Sierra Leone & & 0.30 & 0.36 & 0.28 & 0.33 & 0.47 & 0.60 & 0.31 & 0.40 & 0.39 & 0.48 & 0.29 & 0.36 \\
\hline Tanzania & & 0.62 & 0.81 & 0.53 & 0.69 & 0.63 & 0.83 & 0.49 & 0.65 & 0.63 & 0.82 & 0.51 & 0.67 \\
\hline Tajikistan & & 1.27 & 1.71 & 0.88 & 1.19 & 0.32 & 0.41 & 0.24 & 0.31 & 0.79 & 1.06 & 0.56 & 0.75 \\
\hline Togo & & 0.30 & 0.36 & 0.28 & 0.33 & 0.47 & 0.60 & 0.31 & 0.40 & 0.39 & 0.48 & 0.29 & 0.36 \\
\hline Uganda & & 0.89 & 1.15 & 0.60 & 0.78 & 0.54 & 0.68 & 0.32 & 0.41 & 0.71 & 0.91 & 0.46 & 0.60 \\
\hline Uzbekistan & & 1.27 & 1.71 & 0.88 & 1.19 & 0.32 & 0.41 & 0.24 & 0.31 & 0.79 & 1.06 & 0.56 & 0.75 \\
\hline Viet nam & & 1.06 & 1.37 & 0.53 & 0.69 & 0.98 & 1.33 & 0.71 & 0.96 & 1.02 & 1.35 & 0.62 & 0.83 \\
\hline Zambia & & 0.57 & 0.78 & 0.35 & 0.48 & 0.60 & 0.75 & 0.40 & 0.51 & 0.59 & 0.77 & 0.38 & 0.49 \\
\hline Zimbabwe & & 0.97 & 1.30 & 0.67 & 0.90 & 1.10 & 1.49 & 0.50 & 0.68 & 1.04 & 1.40 & 0.58 & 0.79 \\
\hline mean & & 0.63 & 0.82 & 0.47 & 0.61 & 0.64 & 0.85 & 0.42 & 0.56 & 0.63 & 0.83 & 0.45 & 0.59 \\
\hline median & & 0.30 & 0.36 & 0.28 & 0.33 & 0.55 & 0.68 & 0.32 & 0.41 & 0.48 & 0.62 & 0.39 & 0.52 \\
\hline stdev & & 0.46 & 0.65 & 0.29 & 0.41 & 0.38 & 0.54 & 0.24 & 0.33 & 0.37 & 0.52 & 0.23 & 0.32 \\
\hline
\end{tabular}


Table 2. Export Supply Elasticities, cont'd

GTAP2001 GTAP2004

GTAP2004

Average 2001-2004

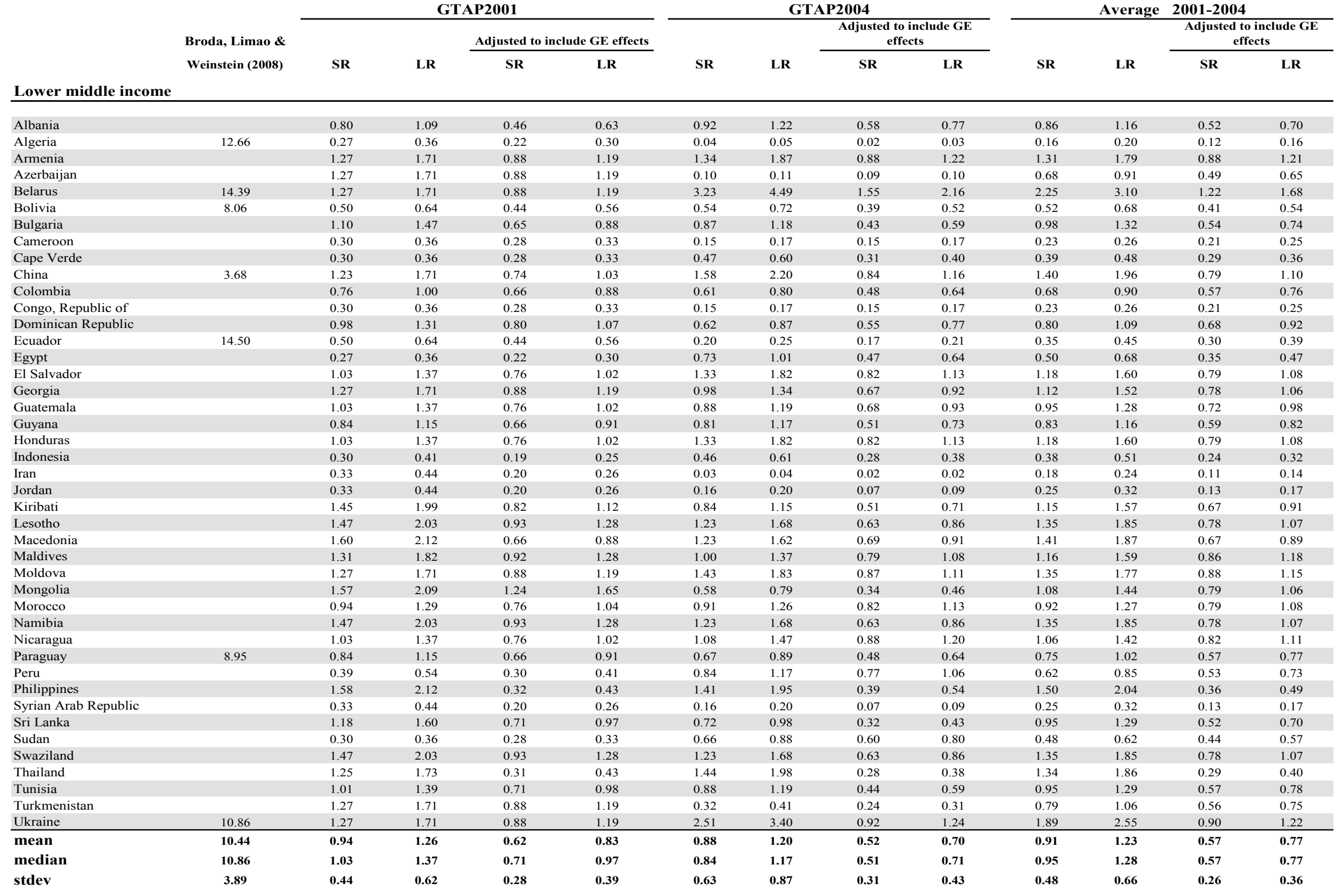


Table 2. Export Supply Elasticities, cont'd GTAP2001

GTAP2004 Adjusted to include GE

Broda, Limao \&

Adjusted to include GE effects effects

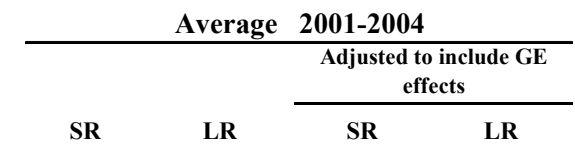

$\underline{\text { Upper middle income }}$

Weinstein (2008)

SR

LR

LR

SR LR

SR

LR

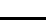

\begin{tabular}{|c|c|c|c|c|c|c|c|c|c|c|c|c|c|}
\hline Argentina & & 0.69 & 0.92 & 0.58 & 0.77 & 0.53 & 0.68 & 0.40 & 0.52 & 0.61 & 0.80 & 0.49 & 0.64 \\
\hline Belize & & 1.03 & 1.37 & 0.76 & 1.02 & 1.33 & 1.82 & 0.82 & 1.13 & 1.18 & 1.60 & 0.79 & 1.08 \\
\hline Botswana & & 1.43 & 1.98 & 1.05 & 1.45 & 0.20 & 0.25 & 0.18 & 0.23 & 0.82 & 1.12 & 0.62 & 0.84 \\
\hline Brazil & & 0.93 & 1.29 & 0.68 & 0.95 & 0.71 & 0.99 & 0.49 & 0.68 & 0.82 & 1.14 & 0.58 & 0.81 \\
\hline Chile & & 0.51 & 0.68 & 0.36 & 0.48 & 0.47 & 0.66 & 0.32 & 0.44 & 0.49 & 0.67 & 0.34 & 0.46 \\
\hline Costa Rica & & 1.03 & 1.37 & 0.76 & 1.02 & 0.81 & 1.08 & 0.32 & 0.43 & 0.92 & 1.23 & 0.54 & 0.72 \\
\hline Croatia & & 2.17 & 2.98 & 1.51 & 2.07 & 1.76 & 2.48 & 1.05 & 1.47 & 1.97 & 2.73 & 1.28 & 1.77 \\
\hline Dominica & & 0.98 & 1.31 & 0.80 & 1.07 & 0.62 & 0.87 & 0.55 & 0.77 & 0.80 & 1.09 & 0.68 & 0.92 \\
\hline Gabon & & 0.30 & 0.36 & 0.28 & 0.33 & 0.15 & 0.17 & 0.15 & 0.17 & 0.23 & 0.26 & 0.21 & 0.25 \\
\hline Grenada & & 0.98 & 1.31 & 0.80 & 1.07 & 0.62 & 0.87 & 0.55 & 0.77 & 0.80 & 1.09 & 0.68 & 0.92 \\
\hline Jamaica & & 0.98 & 1.31 & 0.80 & 1.07 & 0.62 & 0.87 & 0.55 & 0.77 & 0.80 & 1.09 & 0.68 & 0.92 \\
\hline Kazakhstan & & 1.27 & 1.71 & 0.88 & 1.19 & 0.72 & 0.96 & 0.46 & 0.61 & 0.99 & 1.33 & 0.67 & 0.90 \\
\hline Latvia & 15.06 & 2.59 & 3.52 & 1.66 & 2.25 & 1.45 & 1.99 & 0.98 & 1.35 & 2.02 & 2.75 & 1.32 & 1.80 \\
\hline Lebanon & 23.62 & 0.33 & 0.44 & 0.20 & 0.26 & 0.16 & 0.20 & 0.07 & 0.09 & 0.25 & 0.32 & 0.13 & 0.17 \\
\hline Libya & & 0.27 & 0.36 & 0.22 & 0.30 & 0.04 & 0.05 & 0.02 & 0.03 & 0.16 & 0.20 & 0.12 & 0.16 \\
\hline Lithuania & 16.68 & 1.62 & 2.20 & 0.84 & 1.15 & 2.05 & 2.84 & 0.68 & 0.95 & 1.83 & 2.52 & 0.76 & 1.05 \\
\hline Malaysia & & 1.09 & 1.49 & 0.49 & 0.68 & 1.17 & 1.61 & 0.55 & 0.76 & 1.13 & 1.55 & 0.52 & 0.72 \\
\hline Mauritius & & 0.42 & 0.54 & 0.25 & 0.32 & 1.33 & 1.80 & 0.55 & 0.74 & 0.87 & 1.17 & 0.40 & 0.53 \\
\hline Mexico & & 0.63 & 0.87 & 0.25 & 0.35 & 1.10 & 1.53 & 0.26 & 0.37 & 0.87 & 1.20 & 0.26 & 0.36 \\
\hline Panama & & 1.03 & 1.37 & 0.76 & 1.02 & 0.94 & 1.27 & 0.74 & 1.00 & 0.99 & 1.32 & 0.75 & 1.01 \\
\hline Poland & & 1.66 & 2.31 & 1.34 & 1.86 & 1.04 & 1.43 & 0.57 & 0.79 & 1.35 & 1.87 & 0.96 & 1.32 \\
\hline Romania & & 1.12 & 1.55 & 0.58 & 0.79 & 2.13 & 2.90 & 1.21 & 1.64 & 1.63 & 2.22 & 0.89 & 1.22 \\
\hline Russian Federation & 9.17 & 0.30 & 0.39 & 0.24 & 0.32 & 0.22 & 0.28 & 0.16 & 0.20 & 0.26 & 0.34 & 0.20 & 0.26 \\
\hline Seychelles & & 0.42 & 0.54 & 0.25 & 0.32 & 0.66 & 0.88 & 0.60 & 0.80 & 0.54 & 0.71 & 0.43 & 0.56 \\
\hline South Africa & & 0.96 & 1.35 & 0.67 & 0.93 & 0.95 & 1.30 & 0.60 & 0.83 & 0.95 & 1.32 & 0.63 & 0.88 \\
\hline St. Kitts and Nevis & & 0.98 & 1.31 & 0.80 & 1.07 & 0.62 & 0.87 & 0.55 & 0.77 & 0.80 & 1.09 & 0.68 & 0.92 \\
\hline St. Lucia & & 0.98 & 1.31 & 0.80 & 1.07 & 0.62 & 0.87 & 0.55 & 0.77 & 0.80 & 1.09 & 0.68 & 0.92 \\
\hline St. Vincent \& Grens. & & 0.98 & 1.31 & 0.80 & 1.07 & 0.62 & 0.87 & 0.55 & 0.77 & 0.80 & 1.09 & 0.68 & 0.92 \\
\hline Suriname & & 0.84 & 1.15 & 0.66 & 0.91 & 0.81 & 1.17 & 0.51 & 0.73 & 0.83 & 1.16 & 0.59 & 0.82 \\
\hline Turkey & & 0.77 & 1.05 & 0.54 & 0.73 & 0.59 & 0.81 & 0.35 & 0.48 & 0.68 & 0.93 & 0.44 & 0.61 \\
\hline Uruguay & & 0.72 & 0.99 & 0.54 & 0.74 & 1.23 & 1.66 & 0.62 & 0.83 & 0.98 & 1.33 & 0.58 & 0.78 \\
\hline Venezuela & & 0.12 & 0.14 & 0.08 & 0.10 & 0.10 & 0.12 & 0.06 & 0.07 & 0.11 & 0.13 & 0.07 & 0.08 \\
\hline$\overline{\text { mean }}$ & 16.13 & 0.94 & 1.27 & 0.66 & 0.90 & 0.83 & 1.13 & 0.50 & 0.69 & 0.88 & 1.20 & 0.58 & 0.79 \\
\hline median & 15.87 & 0.98 & 1.31 & 0.67 & 0.94 & 0.69 & 0.92 & 0.55 & 0.77 & 0.82 & 1.13 & 0.60 & 0.83 \\
\hline stdev & 5.94 & 0.54 & 0.74 & 0.37 & 0.51 & 0.53 & 0.74 & 0.28 & 0.39 & 0.48 & 0.67 & 0.29 & 0.41 \\
\hline
\end{tabular}


Table 2. Export Supply Elasticities, cont'd GTAP2001

GTAP2004

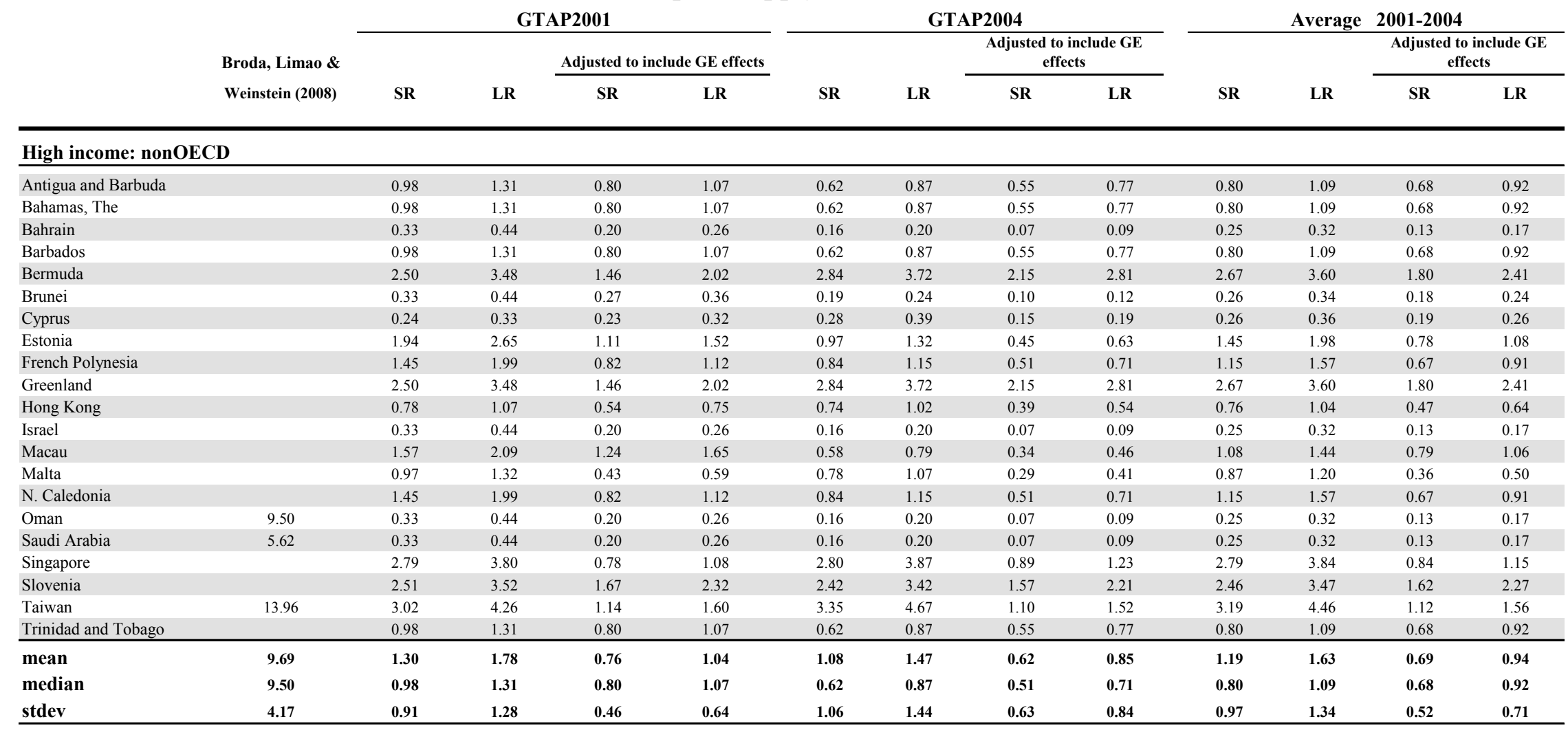


Table 2. Export Supply Elasticities, concluded

GTAP2001 GTAP2004

\begin{tabular}{|c|c|c|c|c|c|c|c|c|c|c|c|c|c|}
\hline & \multirow{3}{*}{$\begin{array}{l}\text { Broda, Limao \& } \\
\text { Weinstein (2008) }\end{array}$} & \multicolumn{4}{|c|}{ GTAP2001 } & \multicolumn{4}{|c|}{ GTAP2004 } & \multicolumn{2}{|c|}{ Average } & \multicolumn{2}{|c|}{ 2001-2004 } \\
\hline & & \multirow[b]{2}{*}{ SR } & \multirow[b]{2}{*}{ LR } & \multicolumn{2}{|c|}{ Adjusted to include GE effects } & \multirow[b]{2}{*}{ SR } & \multirow[b]{2}{*}{ LR } & \multicolumn{2}{|c|}{$\begin{array}{c}\begin{array}{c}\text { Adjusted to include GE } \\
\text { effects }\end{array} \\
\end{array}$} & \multirow[b]{2}{*}{ SR } & \multirow[b]{2}{*}{ LR } & \multicolumn{2}{|c|}{$\begin{array}{c}\begin{array}{c}\text { Adjusted to include GE } \\
\text { effects }\end{array} \\
\end{array}$} \\
\hline & & & & SR & LR & & & SR & LR & & & SR & LR \\
\hline \multicolumn{14}{|l|}{$\begin{array}{l}\text { High income: OECD } \\
\end{array}$} \\
\hline Australia & & 0.56 & 0.72 & 0.48 & 0.63 & 0.64 & 0.87 & 0.57 & 0.77 & 0.60 & 0.80 & 0.53 & 0.70 \\
\hline Austria & & 1.46 & 2.00 & 0.92 & 1.24 & 1.53 & 2.15 & 0.82 & 1.15 & 1.50 & 2.08 & 0.87 & 1.20 \\
\hline Belgium & & 4.81 & 6.46 & 1.67 & 2.25 & 3.14 & 4.47 & 1.39 & 1.95 & 3.98 & 5.46 & 1.53 & 2.10 \\
\hline Canada & & 1.59 & 2.17 & 1.21 & 1.65 & 1.22 & 1.61 & 0.75 & 0.99 & 1.40 & 1.89 & 0.98 & 1.32 \\
\hline Czech Republic & 10.03 & 1.52 & 2.10 & 0.79 & 1.09 & 1.48 & 2.07 & 0.40 & 0.56 & 1.50 & 2.09 & 0.60 & 0.82 \\
\hline Denmark & & 2.02 & 2.78 & 1.21 & 1.66 & 1.00 & 1.32 & 0.66 & 0.87 & 1.51 & 2.05 & 0.93 & 1.27 \\
\hline Finland & & 2.10 & 3.00 & 0.96 & 1.37 & 1.54 & 2.14 & 0.78 & 1.09 & 1.82 & 2.57 & 0.87 & 1.23 \\
\hline France & & 0.97 & 1.30 & 0.76 & 1.02 & 1.71 & 2.28 & 0.95 & 1.27 & 1.34 & 1.79 & 0.85 & 1.14 \\
\hline Germany & & 3.42 & 4.77 & 2.26 & 3.14 & 2.41 & 3.42 & 1.32 & 1.87 & 2.91 & 4.09 & 1.79 & 2.51 \\
\hline Greece & & 1.77 & 2.38 & 1.11 & 1.50 & 0.59 & 0.79 & 0.56 & 0.76 & 1.18 & 1.59 & 0.84 & 1.13 \\
\hline Hungary & & 1.27 & 1.74 & 0.61 & 0.82 & 1.81 & 2.50 & 0.68 & 0.93 & 1.54 & 2.12 & 0.64 & 0.88 \\
\hline Iceland & & 0.53 & 0.71 & 0.34 & 0.46 & 1.97 & 2.69 & 1.00 & 1.37 & 1.25 & 1.70 & 0.67 & 0.91 \\
\hline Ireland & & 1.86 & 2.50 & 0.84 & 1.13 & 1.28 & 1.72 & 0.41 & 0.56 & 1.57 & 2.11 & 0.62 & 0.84 \\
\hline Italy & & 1.27 & 1.75 & 0.84 & 1.16 & 1.20 & 1.66 & 0.59 & 0.82 & 1.24 & 1.70 & 0.72 & 0.99 \\
\hline Japan & & 1.54 & 2.18 & 1.25 & 1.76 & 1.60 & 2.26 & 1.20 & 1.69 & 1.57 & 2.22 & 1.22 & 1.72 \\
\hline Korea & & 2.45 & 3.48 & 0.79 & 1.11 & 2.22 & 3.13 & 0.67 & 0.94 & 2.34 & 3.31 & 0.73 & 1.02 \\
\hline Luxembourg & & 3.83 & 5.14 & 2.15 & 2.88 & 2.01 & 2.77 & 1.75 & 2.42 & 2.92 & 3.95 & 1.95 & 2.65 \\
\hline Netherlands & & 2.23 & 3.06 & 1.02 & 1.40 & 1.78 & 2.41 & 0.51 & 0.69 & 2.01 & 2.73 & 0.77 & 1.04 \\
\hline New Zealand & & 1.07 & 1.45 & 0.76 & 1.03 & 0.95 & 1.31 & 0.73 & 1.00 & 1.01 & 1.38 & 0.74 & 1.01 \\
\hline Norway & & 0.53 & 0.71 & 0.34 & 0.46 & 0.22 & 0.27 & 0.17 & 0.21 & 0.37 & 0.49 & 0.26 & 0.33 \\
\hline Portugal & & 1.72 & 2.37 & 1.25 & 1.73 & 1.57 & 2.16 & 1.14 & 1.57 & 1.64 & 2.26 & 1.20 & 1.65 \\
\hline Slovakia & & 1.93 & 2.71 & 0.68 & 0.94 & 1.66 & 2.32 & 0.53 & 0.73 & 1.80 & 2.51 & 0.60 & 0.84 \\
\hline Spain & & 0.95 & 1.32 & 0.74 & 1.03 & 1.22 & 1.63 & 0.85 & 1.13 & 1.08 & 1.47 & 0.80 & 1.08 \\
\hline Sweden & & 2.66 & 3.72 & 1.61 & 2.24 & 2.08 & 2.94 & 1.02 & 1.44 & 2.37 & 3.33 & 1.32 & 1.84 \\
\hline Switzerland & & 1.36 & 1.82 & 1.06 & 1.42 & 1.33 & 1.84 & 0.80 & 1.11 & 1.35 & 1.83 & 0.93 & 1.27 \\
\hline United States of America & & 1.63 & 2.22 & 1.41 & 1.93 & 1.49 & 2.05 & 1.16 & 1.60 & 1.56 & 2.14 & 1.29 & 1.77 \\
\hline United Kingdom & & 1.47 & 1.91 & 1.37 & 1.79 & 0.83 & 1.06 & 0.74 & 0.95 & 1.15 & 1.48 & 1.06 & 1.37 \\
\hline mean & 10.03 & 1.81 & 2.48 & 1.04 & 1.43 & 1.53 & 2.11 & 0.82 & 1.13 & 1.67 & 2.29 & 0.93 & 1.28 \\
\hline median & 10.03 & 1.61 & 2.20 & 0.94 & 1.30 & 1.54 & 2.15 & 0.76 & 1.04 & 1.53 & 2.10 & 0.84 & 1.14 \\
\hline stdev & - & 1.00 & 1.37 & 0.48 & 0.66 & 0.61 & 0.87 & 0.35 & 0.50 & 0.75 & 1.05 & 0.39 & 0.55 \\
\hline \multicolumn{14}{|l|}{ Overall } \\
\hline Mean & 11.85 & 1.04 & 1.41 & 0.68 & 0.91 & 0.93 & 1.27 & 0.55 & 0.75 & 0.99 & 1.34 & 0.62 & 0.83 \\
\hline Median & 11.35 & 0.98 & 1.31 & 0.68 & 0.94 & 0.76 & 1.03 & 0.51 & 0.73 & 0.83 & 1.16 & 0.59 & 0.82 \\
\hline Stdev & 5.08 & 0.75 & 1.04 & 0.41 & 0.57 & 0.68 & 0.95 & 0.37 & 0.51 & 0.67 & 0.94 & 0.36 & 0.50 \\
\hline
\end{tabular}

Note1: Income group: Economies are divided according to 2007 GNI per capita, calculated using the World Bank

Atlas method. The groups are: low income, \$935 or less; lower middle income, \$936-\$3,705; upper middle income,

\$3,706 - \$11,455; and high income, \$11,456 or more. Source: World Bank

Note2: from Broda, Limao Weinstein (2008) 


\section{Computing Elasticities of a Country's Trade Balance With Respect to a Change in The EXChange RATE}

Trade elasticities are frequently needed to answer many policy questions facing countries and one such issue is reaching a judgement whether a country's real exchange rate is in equilibrium This section derives an analytical expression for how a change in a country's real exchange rate would affect its trade balance and shows how the elasticities estimated in previous section can be used in this exercise.

\section{A. The Trade Balance and the Real Exchange Rate}

A country's trade balance, in terms of foreign currency, can be written:

$$
T B^{*}=P_{E}^{*} E-P_{M}^{*} M
$$

where $P_{E}^{*}$ is the price of exports in foreign currency, $P_{M}^{*}$ is the price of imports in foreign currency, $\mathrm{E}$ is the volume of exports, $\mathrm{M}$ is the volume of imports, and $T B^{*}$ is the trade balance. Note that $\mathrm{E}$ and $\mathrm{M}$ are functions of both the domestic and foreign prices of each good. Totally differentiating equation (34) gives:

$$
d T B^{*}=P_{E}^{*} E\left(\hat{p}_{E}^{*}+\hat{E}\right)-P_{M}^{*} M\left(\hat{p}_{M}^{*}+\hat{M}\right)
$$

where $^{\wedge}$ denotes proportional change, i.e. $\hat{M}=\frac{d M}{M}$. The domestic prices of imports and exports are related to foreign prices and the nominal exchange rate, $\mathrm{r}$ :

$$
\begin{array}{ll}
p_{M} r=p_{M}^{*}, \text { or } & \hat{p}_{M}=\hat{p}_{M}^{*}-\hat{r} \\
p_{E} r=p_{E}^{*}, \text { or } & \hat{p}_{E}=\hat{p}_{E}^{*}-\hat{r} .
\end{array}
$$

To allow for the possibility that changes in foreign prices or the exchange rate are not fully passed through into domestic prices, the two equations above can be modified to include pass-through coefficients. For example:

$$
\begin{gathered}
\hat{p}_{M}=\phi_{M}\left(\hat{p}_{M}^{*}-\hat{r}\right) \\
\hat{p}_{E}=\phi_{E}\left(\hat{p}_{E}^{*}-\hat{r}\right)
\end{gathered}
$$

where $\phi_{M}$ and $\phi_{E}$ are the pass-though coefficients for import and export prices respectively and lie between zero and one. If $\phi_{M}=\phi_{E}=1$, then pass through is complete and changes in foreign prices are fully reflected in domestic prices. There is no consensus in the literature on 
values for these parameters. Frankel, Parsley, and Wei (2005) estimate that for developing countries and emerging markets, the pass-through coefficient is in the range of 0.66 to 0.77 .

In equation (35), expressions for changes in foreign prices and quantities are needed. Define the following:

$\hat{E}^{D}=\eta_{X} \hat{p}_{E}^{*} \quad\left(\right.$ export demand equation, with export demand elasticity $\left.\eta_{X}<0\right)$

$\hat{E}^{S}=\varepsilon_{X} \hat{p}_{E} \quad$ (export supply equation, with export supply elasticity $\varepsilon_{X}>0$ )

$\hat{M}^{D}=\eta_{M} \hat{p}_{M} \quad$ (import demand equation, with import demand elasticity $\eta_{M}<0$ )

$\hat{M}^{S}=\varepsilon_{M} \hat{p}_{M}^{*} \quad$ (import supply equation, with import supply elasticity $\left.\varepsilon_{M}>0\right)$.

In these equations, export demand and import supply depend on foreign prices, while import demand and export supply depend on domestic prices. In the export market, export demand must equal export supply $\left(\hat{E}^{D}=\hat{E}^{S}\right): \eta_{X} \hat{p}_{E}^{*}=\varepsilon_{X} \hat{p}_{E}$. Using equation (37), the solution for $\hat{p}_{E}^{*}$ is:

$$
\hat{p}_{E}^{*}=\frac{-\varepsilon_{X} \phi_{E}}{\left(\eta_{X}-\phi_{E} \varepsilon_{X}\right)} \hat{r}
$$

Following a similar procedure to solve for $\hat{p}_{M}^{*}$ :

$$
\hat{p}_{M}^{*}=\frac{\eta_{M} \phi_{M}}{\left(\phi_{M} \eta_{M}-\varepsilon_{M}\right)} \hat{r}
$$

Note that choices of elasticity values determine the response of $\hat{p}_{E}^{*}$ to a change in $r$. For example, if the country under consideration is "small", then $\eta_{X}=\infty$ and $\hat{p}_{E}^{*}=0$ from equation (38). Also, "small" implies that the import supply elasticity is infinite. From equation (39), $\varepsilon_{M}=\infty$ implies that $\hat{p}_{M}^{*}=0$ from equation (39). So, a small country is unable to influence the foreign currency prices of exports and imports.

Substituting equations (38) and (39) into equation (35), along with the solutions for $\hat{E}$ and $\hat{M}$, gives:

$$
\frac{d T B^{*}}{d r / r}=P_{E}^{*} E\left[\frac{-\varepsilon_{X} \phi_{E}\left(\eta_{X}+1\right)}{\eta_{X}-\varepsilon_{X} \phi_{E}}\right]-P_{M}^{*} M\left[\frac{\eta_{M} \phi_{M}\left(1+\varepsilon_{M}\right)}{\eta_{M} \phi_{M}-\varepsilon_{M}}\right]
$$

Dividing both sides of equation (40) by GDP (denominated in the foreign currency) gives: 


$$
\frac{d T B^{*} / G D P^{*}}{d r / r}=s_{X}\left[\frac{-\varepsilon_{X} \phi_{E}\left(\eta_{X}+1\right)}{\eta_{X}-\varepsilon_{X} \phi_{E}}\right]-s_{M}\left[\frac{\eta_{M} \phi_{M}\left(1+\varepsilon_{M}\right)}{\eta_{M} \phi_{M}-\varepsilon_{M}}\right]
$$

where $s_{X}$ and $s_{M}$ are the shares of exports and imports in GDP respectively. To compute how the trade balance would change, denominated in domestic currency, use the relationship:

$$
T B r=T B^{*}
$$

where TB is the trade balance measured in domestic currency.

Differentiating equation (42) with respect to $r$, gives:

$$
\frac{d T B}{d r} r=\frac{d T B^{*}}{d r}-T B
$$

or,

$$
\frac{d T B}{d r} r=\frac{1}{r} \frac{d T B^{*}}{d r / r}-T B
$$

Substituting equation (40) into equation (44) and manipulating, gives:

$$
\frac{d T B / G D P}{d r / r}=s_{X}\left[\frac{-\eta_{X}\left(1+\varepsilon_{X} \phi_{E}\right)}{\eta_{X}-\varepsilon_{X} \phi_{E}}\right]-s_{M}\left[\frac{\varepsilon_{M}\left(1+\eta_{M} \phi_{M}\right)}{\eta_{M} \phi_{M}-\varepsilon_{M}}\right]
$$

It needs to be emphasized that a devaluation could cause the trade balance to improve measured in foreign currency terms, but deteriorate when measured in domestic currency terms. Consider the small country case, where prices are fixed in foreign currency terms. Suppose a devaluation raises the domestic prices of imports and exports by the full amount of the exchange rate change - the case of full pass through. Then export volume will rise and import volume will fall. Measured at foreign prices, the trade balance must improve because foreign prices are fixed and export volume increased, while import volume decreases. So the change in the trade balance must be positive. Measured at domestic prices, however, the change in the trade balance could be positive or negative. On the export side, the domestic price of exports rises and so does volume, so export revenue must increase. On the import side, however, expenditure on imports could rise or fall. This is because the domestic price of imports rises with the devaluation, but the volume falls. So what happens to import expenditure depends on the elasticity of demand for imports, as shown in equation (45) - it will depend on whether $\eta_{M}$ is greater or less than one. Using equation (43) it is easy to see that the only situation in which the trade balance must change in the same direction in both foreign and domestic currency is if the trade balance is initially zero. 


\section{B. Special Cases}

Equations (41) and (45) are general formulas that show how a change in a country's real exchange rate affects its trade balance. Under certain assumptions about elasticity values, these formulas reduce to simpler forms. The next section examines a number of specific cases, including the IMF's assessment of country exchange rates in the context of the Consultative Group on Exchange Rates (CGER) exercise.

Case 1: Small Country $\left(\eta_{X}=\infty, \varepsilon_{M}=\infty\right)$

Many low-income countries are "small" in world markets for their imports and exports, that is, they are unable to affect prices denominated in foreign currency by how much they buy or sell. Under these assumptions, the export demand elasticity that they face and import supply elasticity would be infinite. In this case, letting $\eta_{X}=\varepsilon_{M}=\infty$, equation (41) reduces to a simple form:

$$
\frac{\partial T B^{*} / G D P^{*}}{d r / r}=-S_{X} \varepsilon_{X} \phi_{E}+s_{M} \eta_{M} \phi_{M}<0
$$

where $\varepsilon_{X}$ is a country's export supply elasticity (defined as a positive number) and $\eta_{M}$ is the

import demand elasticity (defined as a negative number). Given that the shares of exports and imports in GDP are positive, equation (46) must be negative. That is, under the "small country" assumption, an appreciation of the real exchange rate must worsen the trade balance (or alternatively, a depreciation of the real exchange rate will improve the trade balance). A depreciation raises the domestic currency price of exports (while the foreign price remains unchanged), causing producers to increase the quantity of exports supplied. It also raises the domestic currency price of imports (while the foreign price remains unchanged), causing consumers to reduce the quantity of imports demanded. Both of these effects work to improve the trade balance. Table 1 of this paper presented estimated values for export supply $\varepsilon_{X}$ and import demand elasticities $\eta_{M}$. Using these elasticity values, together with equation (46), it is possible to calculate how a given change in a country's exchange rate would affect its trade balance.

Case 2: CGER Assumptions $\left(\varepsilon_{X}=\infty, \varepsilon_{M}=\infty\right)$

Under the basic set of CGER assumptions, both the supply of exports and imports are assumed to be perfectly elastic - the so called "Keynesian Case" in the literature. Under these assumptions, equation (45) reduces to: 


$$
\frac{\partial T B / G D P}{d r / r}=s_{X} \eta_{X}+s_{M}\left(1+\eta_{M} \phi_{M}\right)<0^{1}
$$

Equation (47) is frequently referred to as the "Marshall-Lerner" condition, which is the condition for a depreciation to improve the trade balance in the special case where supply elasticities are assumed to be infinite and $\phi_{E}=\phi_{M}=1$ (full pass-through). Therefore, under these assumptions, a depreciation will improve the trade balance when trade is balanced initially $\left(s_{X}=s_{M}\right.$ ) if the sum of the export and import demand elasticities is greater than one. Thus, the CGER note presents the conditions that must hold for a depreciation to improve the trade balance, using the assumptions that the elasticities of import and export supply are infinite.

In summary, the CGER exercise assumes that both export and import supply elasticities are infinite, while the "small country" assumption assumes that the export demand and import supply elasticities are infinite. This difference reflects alternative assumptions about pricing behavior in countries, namely, whether producers set prices in dome or foreign currency. If countries are unable to affect the foreign prices of the goods that they import and export, then it would be appropriate to use the "small country" assumptions with regard to elasticities. If the countries under study have some market power, i.e., are able to influence foreign prices by how much they sell, then it would be appropriate to use an export demand elasticity that is less than infinity. A consequence of using the "small country" assumptions is that a real devaluation will always improve the trade balance. This is because a devaluation cannot reduce the foreign-currency prices of imports and exports, so there the devaluation only affects trade volumes - export volume rises and import volume declines. In the basic CGER analysis, a devaluation will in general have ambiguous effects on the trade balance in domestic currency terms. Table 3 below summarizes the cases described above and presents the conditions for a devaluation to improve the trade balance.

\footnotetext{
${ }^{1}$ The corresponding condition that appears in the CGER note "Adjusting Elasticities For Commodity Exporters," is: $\frac{d T B / G D P}{d r / r}=s_{X} \eta_{X}+s_{M}\left(1-\eta_{M}\right)$. This differs from equation (42) because in the CGER, $\eta_{M}$ is defined with respect to changes in the RER, so its sign is positive. In this note, $\eta_{M}$ is defined with respect to changes in import prices, so its sign is negative.
} 
Table 3. Summary of How changes in the Exchange Rate Affect the Trade Balance

Small Country

$\eta_{X}=-\infty$

$\varepsilon_{X}>0$

$\eta_{M}<0$

$\varepsilon_{M}=\infty$

Condition for devaluation to

improve trade balance in foreign

currency
CGER, Main Assumptions 1/

$$
\begin{aligned}
& \eta_{X}=-0.71 \\
& \varepsilon_{X}=\infty \\
& \eta_{M}=-0.92 \\
& \varepsilon_{M}=\infty
\end{aligned}
$$

$$
s_{X}\left(\eta_{X}+1\right)+s_{M} \eta_{M} \phi_{M}<0
$$

$$
-s_{X} \varepsilon_{X} \phi_{E}+s_{M} \eta_{M} \phi_{M}<0
$$

$$
s_{X} \eta_{X}+s_{M}\left(1+\eta_{M} \phi_{M}\right)<0
$$

\section{General Case}

$\eta_{X}<0$

$\varepsilon_{X}>0$

$\eta_{M}<0$

$\varepsilon_{M}>0$

$s_{X}\left[\frac{-\varepsilon_{X} \phi_{E}\left(\eta_{X}+1\right)}{\eta_{X}-\varepsilon_{X} \phi_{E}}\right]-$

$s_{M}\left[\frac{\eta_{M} \phi_{M}\left(1+\varepsilon_{M}\right)}{\eta_{M} \phi_{M}-\varepsilon_{M}}\right]<0$

$s_{X}\left[\frac{-\eta_{X}\left(1+\varepsilon_{X} \phi_{E}\right)}{\eta_{X}-\varepsilon_{X} \phi_{E}}\right]-$

$s_{M}\left[\frac{\varepsilon_{M}\left(1+\eta_{M} \phi_{M}\right)}{\eta_{M} \phi_{M}-\varepsilon_{M}}\right]<0$

\section{currency \\ Condition for devaluation to}

Source: Author's calculations.

Note: (i) $\eta_{x}$ is the export demand elasticity; (ii) $\varepsilon_{s}$ is the export supply elasticity; (iii) $\eta_{m}$ is the import demand elasticity; (iv) $\varepsilon_{m}$ is the import supply elasticity; (v) $\phi_{E}$

measures the extent of pass through into export prices; (vi) $\phi_{M}$ measures the extent of pass through into import prices; and (vii) $S_{x}$ and $S_{M}$ are the shares of exports and imports in GDP.

1/ The CGER employs a number of exceptions to this general rule. Regarding import demand elasticities $\left(\eta_{m}\right)$, the CGER uses a value of -1.0 for the euro area, United States,

Malaysia, and Columbia; a value of -0.67 for China; and a value of -1.10 for Brazil. Regarding export demand elasticities, $\left(\eta_{x}\right)$, the CGER uses -0.85 for the United States and Brazil, -0.95 for china, and -0.94 for Malaysia. The CGER also adjusts the overall export elasticities depending on its share of commodity exports and uses an elasticity of -1.0 for these. 
Table 4 reports the trade balance elasticities that result from using various formulae. In this table, the column labelled "CGER" refers to the trade balance elasticities that result from using the main CGER assumptions noted in table 3. Note that for about five countries and regions, the CGER uses elasticities that differ from -0.71 and 0.92 and these are noted in the footnote accompanying Table 3 . The column labelled "small country" lists the trade balance elasticities that result from assuming that import supply and export demand elasticities are infinite. In addition, these trade balance elasticities also use the import demand and export supply elasticities reported in tables 1 and 2 respectively, from the columns labelled long-run general equilibrium elasticities - the column on the far right of tables 1 and 2 labelled "LR, adjusted to include GE effects."

The column labelled "general" refers to the trade balance elasticities that result from applying the assumptions listed in Table 3. For illustrative purposes, this case assumes that the import supply elasticity is infinite, import demand elasticities are taken from table 1, export supply elasticities are taken from table 2, and the main CGER assumptions regarding export demand elasticities are used. This general formula would be appropriate to use for cases in which any or all of the elasticities are not infinite. This will depend on what the country in question exports and imports and to what extent the country is able to influence world prices by how much it exports or imports.

For the "general" case reported in table 3, any value for export demand elasticities could have selected, but in the absence of more reliable estimates, the main CGER values (-0.71) were used. For a summary of econometric studies on export demand elasticities, see Goldstein and Khan (1985) or Stern, Francois, and Schumacher (1976).

For all the trade balance elasticities reported in Table 4, it was assumed that $\phi_{E}=\phi_{M}=1$, that is, the full pass-through case. Country-specific values could be used if known.

In table 4, for most countries, the absolute value of the trade balance elasticity for the "general case" lies between the elasticities for the CGER case and the small country case. An exception is the case of Bangladesh, where the trade balance elasticity for the general case exceeds the CGER elasticity. Using the formulae for changes in the trade balance in domestic currency terms, this will occur when:

$s_{X}\left[\frac{-\eta_{X}\left(\varepsilon_{X}+1\right)}{\eta_{X}-\varepsilon_{X}}\right]+s_{M}\left(\eta_{M}+1\right)>s_{X} \eta_{X}^{C G E R}+s_{M}\left(1-\eta_{M}^{C G E R}\right)$. Substituting in the relevant values,

this condition can be expressed as:

$\eta_{M}>\frac{s_{X}}{s_{M}}\left[\frac{-.2059}{-.71-\varepsilon_{X}}\right]-0.92$. Since the import demand elasticity for Bangladesh is relatively 
high (-0.33), the above condition is satisfied. However, for most other countries, the import demand elasticity is more negative. Also, a low value for $\left(\frac{s_{X}}{s_{M}}\right)$ will increase the likelihood that the above condition is satisfied. In the case of Bangladesh, this ratio is 0.62 . Since the bracketed term in the above condition is positive, a low value for $\left(\frac{s_{X}}{s_{M}}\right)$ means that a low weight will be attached to this term. 
Table 4. Trade Balance Elasticities

(cger, general and small-country assumptions)

\begin{tabular}{lrr}
\multicolumn{3}{c}{ Domestic Currency } \\
\hline & & SMALL \\
CGER & GENERAL & COUNTRY
\end{tabular}

\begin{tabular}{lrr}
\multicolumn{3}{c}{ Foreign currency } \\
\hline & & SMALL \\
CGER & GENERAL & COUNTRY
\end{tabular}

\begin{tabular}{|c|c|c|c|c|c|c|}
\hline \multicolumn{7}{|l|}{ Low income } \\
\hline Bangladesh & -0.14 & 0.06 & -0.42 & -0.28 & -0.07 & -0.56 \\
\hline Benin & -0.06 & -0.18 & -0.23 & -0.16 & -0.28 & -0.33 \\
\hline Burkina Faso & -0.07 & -0.23 & -0.29 & -0.21 & -0.36 & -0.42 \\
\hline Burundi & -0.08 & -0.25 & -0.36 & -0.38 & -0.55 & -0.67 \\
\hline Cambodia & -0.38 & -0.79 & -1.19 & -0.51 & -0.92 & -1.32 \\
\hline Central African Rep. & -0.10 & -0.24 & -0.30 & -0.18 & -0.33 & -0.38 \\
\hline Chad & -0.31 & -0.65 & -0.81 & -0.36 & -0.70 & -0.86 \\
\hline Congo, Dem. Rep. & -0.43 & -0.71 & -0.87 & -0.45 & -0.74 & -0.90 \\
\hline \multicolumn{7}{|l|}{ Comoros } \\
\hline Côte d'Ivoire & -0.29 & -0.60 & -0.81 & -0.28 & -0.58 & -0.79 \\
\hline Eritrea & -0.05 & -0.12 & -0.18 & -0.13 & -0.20 & -0.26 \\
\hline Ethiopia & -0.12 & -0.23 & -0.37 & -0.36 & -0.47 & -0.60 \\
\hline Gambia, The & -0.30 & -0.76 & -1.00 & -0.55 & -1.02 & -1.25 \\
\hline Ghana & -0.20 & -0.52 & -0.68 & -0.39 & -0.71 & -0.87 \\
\hline Guinea & -0.17 & -0.44 & -0.57 & -0.32 & -0.59 & -0.73 \\
\hline Guinea Bissau & -0.18 & -0.45 & -0.59 & -0.33 & -0.61 & -0.74 \\
\hline \multicolumn{7}{|l|}{ Haiti } \\
\hline India & -0.14 & -0.11 & -0.38 & -0.19 & -0.16 & -0.43 \\
\hline Kenya & -0.15 & -0.29 & -0.46 & -0.24 & -0.39 & -0.56 \\
\hline Kyrgyzstan & -0.42 & -0.64 & -1.23 & -0.70 & -0.92 & -1.51 \\
\hline Lao People's Dem.Rep & -0.19 & -0.25 & -0.45 & -0.22 & -0.28 & -0.48 \\
\hline Madagascar & -0.22 & -0.41 & -0.77 & -0.25 & -0.43 & -0.80 \\
\hline Malawi & -0.15 & -0.19 & -0.38 & -0.37 & -0.42 & -0.61 \\
\hline Mali & -0.13 & -0.31 & -0.40 & -0.20 & -0.37 & -0.47 \\
\hline Mauritania & -0.27 & -0.59 & -0.79 & -0.32 & -0.64 & -0.84 \\
\hline \multicolumn{7}{|l|}{ Myanmar } \\
\hline Mozambique & -0.17 & -0.14 & -0.51 & -0.28 & -0.26 & -0.62 \\
\hline Nepal & -0.07 & -0.08 & -0.27 & -0.27 & -0.27 & -0.46 \\
\hline Niger & -0.12 & -0.35 & -0.44 & -0.30 & -0.54 & -0.63 \\
\hline Nigeria & -0.36 & -0.60 & -0.72 & -0.19 & -0.43 & -0.55 \\
\hline Pakistan & -0.08 & -0.08 & -0.22 & -0.16 & -0.16 & -0.30 \\
\hline Papua New Guinea & -0.40 & -0.42 & -1.10 & -0.44 & -0.46 & -1.14 \\
\hline Rwanda & -0.06 & -0.17 & -0.25 & -0.25 & -0.36 & -0.44 \\
\hline Senegal & -0.15 & -0.36 & -0.51 & -0.34 & -0.56 & -0.71 \\
\hline Sierra Leone & -0.16 & -0.37 & -0.49 & -0.24 & -0.46 & -0.58 \\
\hline Tajikistan & -0.08 & -0.28 & -0.44 & -0.49 & -0.68 & -0.85 \\
\hline Tanzania & -0.18 & -0.22 & -0.47 & -0.35 & -0.40 & -0.64 \\
\hline Togo & -0.32 & -0.81 & -1.06 & -0.56 & -1.05 & -1.30 \\
\hline Uganda & -0.12 & -0.09 & -0.25 & -0.28 & -0.25 & -0.41 \\
\hline Uzbekistan & -0.27 & -0.43 & -0.81 & -0.21 & -0.37 & -0.75 \\
\hline Viet nam & -0.57 & -1.69 & -2.60 & -0.71 & -1.83 & -2.75 \\
\hline Zambia & -0.17 & -0.28 & -0.45 & -0.21 & -0.33 & -0.49 \\
\hline \multicolumn{7}{|l|}{ Zimbabwe } \\
\hline mean & -0.20 & -0.39 & -0.62 & -0.32 & -0.52 & -0.74 \\
\hline median & -0.17 & -0.31 & -0.47 & -0.28 & -0.43 & -0.63 \\
\hline stdev & 0.12 & 0.31 & 0.43 & 0.14 & 0.32 & 0.44 \\
\hline
\end{tabular}


Table 4 Continued

\begin{tabular}{crr}
\multicolumn{3}{c}{ Domestic Currency } \\
\hline CGER & GENERAL & COUNTRY
\end{tabular}

\begin{tabular}{ccc}
\multicolumn{3}{c}{ Foreign currency } \\
\hline CGER & GENERAL & COUNTRY
\end{tabular}

\begin{tabular}{|c|c|c|c|c|c|c|}
\hline Lower middle income & & & & & & \\
\hline Albania & -0.17 & -0.19 & -0.44 & -0.39 & -0.42 & -0.66 \\
\hline Algeria & -0.33 & -0.60 & -0.71 & -0.20 & -0.46 & -0.57 \\
\hline Armenia & -0.11 & -0.24 & -0.53 & -0.34 & -0.46 & -0.76 \\
\hline Azerbaijan & -0.40 & -0.85 & -1.33 & -0.23 & -0.69 & -1.17 \\
\hline Belarus & -0.42 & -0.26 & -1.54 & -0.46 & -0.30 & -1.58 \\
\hline Bolivia & -0.23 & -0.34 & -0.58 & -0.20 & -0.30 & -0.54 \\
\hline Bulgaria & -0.47 & -1.01 & -1.68 & -0.58 & -1.12 & -1.79 \\
\hline Cameroon & -0.14 & -0.31 & -0.38 & -0.18 & -0.34 & -0.42 \\
\hline \multicolumn{7}{|l|}{ Cape Verde } \\
\hline China & -0.33 & -0.31 & -0.85 & -0.23 & -0.22 & -0.75 \\
\hline Colombia & -0.15 & -0.19 & -0.38 & -0.18 & -0.22 & -0.41 \\
\hline Congo, Republic of & -0.59 & -1.10 & -1.40 & -0.41 & -0.93 & -1.22 \\
\hline Dominican Republic & -0.19 & -0.48 & -0.82 & -0.26 & -0.55 & -0.89 \\
\hline Ecuador & -0.23 & -0.52 & -0.70 & -0.25 & -0.54 & -0.72 \\
\hline Egypt & -0.21 & -0.30 & -0.50 & -0.30 & -0.39 & -0.59 \\
\hline El Salvador & -0.16 & -0.29 & -0.64 & -0.35 & -0.49 & -0.84 \\
\hline Georgia & -0.26 & -0.38 & -0.92 & -0.46 & -0.58 & -1.12 \\
\hline Guatemala & -0.15 & -0.28 & -0.57 & -0.28 & -0.41 & -0.70 \\
\hline \multicolumn{7}{|l|}{ Guyana } \\
\hline Honduras & -0.37 & -0.64 & -1.42 & -0.69 & -0.97 & -1.75 \\
\hline Indonesia & -0.19 & -0.27 & -0.40 & -0.18 & -0.26 & -0.38 \\
\hline \multicolumn{7}{|l|}{ Iran } \\
\hline Jordan & -0.37 & -0.66 & -0.81 & -0.72 & -1.01 & -1.16 \\
\hline \multicolumn{7}{|l|}{ Kiribati } \\
\hline Lesotho & -0.38 & -0.21 & -1.03 & -0.76 & -0.59 & -1.41 \\
\hline Macedonia & -0.44 & -0.69 & -1.45 & -0.67 & -0.92 & -1.68 \\
\hline \multicolumn{7}{|l|}{ Maldives } \\
\hline Moldova & -0.49 & -1.87 & -3.01 & -1.15 & -2.53 & -3.67 \\
\hline Mongolia & -0.52 & -0.78 & -1.79 & -0.54 & -0.80 & -1.81 \\
\hline Morocco & -0.25 & -0.49 & -0.99 & -0.34 & -0.58 & -1.08 \\
\hline Namibia & -0.30 & -0.22 & -0.83 & -0.37 & -0.30 & -0.90 \\
\hline Nicaragua & -0.31 & -0.72 & -1.41 & -0.64 & -1.05 & -1.74 \\
\hline Paraguay & -0.48 & -0.72 & -1.43 & -0.52 & -0.77 & -1.47 \\
\hline Peru & -0.15 & -0.20 & -0.41 & -0.17 & -0.22 & -0.43 \\
\hline Philippines & -0.26 & -0.23 & -0.49 & -0.36 & -0.33 & -0.59 \\
\hline Syrian Arab Republic & -0.26 & -0.43 & -0.53 & -0.35 & -0.52 & -0.62 \\
\hline Sri Lanka & -0.15 & -0.19 & -0.41 & -0.25 & -0.29 & -0.51 \\
\hline Sudan & -0.11 & -0.19 & -0.32 & -0.11 & -0.19 & -0.31 \\
\hline Swaziland & -0.49 & -0.34 & -1.33 & -0.70 & -0.55 & -1.55 \\
\hline Thailand & -0.58 & -0.26 & -0.73 & -0.57 & -0.26 & -0.72 \\
\hline Tunisia & -0.40 & -0.99 & -1.58 & -0.42 & -1.01 & -1.60 \\
\hline Turkmenistan & -0.30 & -0.42 & -0.83 & -0.08 & -0.20 & -0.61 \\
\hline Ukraine & -0.32 & -0.35 & -1.08 & -0.45 & -0.48 & -1.21 \\
\hline mean & -0.31 & -0.49 & -0.95 & -0.40 & -0.59 & -1.05 \\
\hline median & -0.30 & -0.34 & -0.82 & -0.36 & -0.48 & -0.86 \\
\hline stdev & 0.14 & 0.34 & 0.55 & 0.22 & 0.42 & 0.64 \\
\hline
\end{tabular}


Table 4 Continued

\begin{tabular}{lrr} 
& Domestic Currency & \\
\hline & & SMALL \\
CGER & GENERAL & COUNTRY
\end{tabular}

\begin{tabular}{crr}
\multicolumn{3}{c}{ Foreign currency } \\
\hline & & SMALL \\
CGER & GENERAL & COUNTRY
\end{tabular}

\begin{tabular}{|c|c|c|c|c|c|c|}
\hline Upper middle income & & & & & & \\
\hline Argentina & -0.14 & -0.28 & -0.45 & -0.13 & -0.27 & -0.44 \\
\hline \multicolumn{7}{|l|}{ Belize } \\
\hline Botswana & -0.35 & -0.52 & -1.05 & -0.24 & -0.41 & -0.94 \\
\hline Brazil & -0.10 & -0.14 & -0.30 & -0.09 & -0.13 & -0.29 \\
\hline Chile & -0.23 & -0.51 & -0.73 & -0.30 & -0.57 & -0.79 \\
\hline Costa Rica & -0.45 & -0.63 & -1.27 & -0.53 & -0.71 & -1.35 \\
\hline Croatia & -0.36 & -0.48 & -1.62 & -0.42 & -0.54 & -1.68 \\
\hline \multicolumn{7}{|l|}{ Dominica } \\
\hline Gabon & -0.49 & -0.83 & -1.07 & -0.18 & -0.53 & -0.77 \\
\hline \multicolumn{7}{|l|}{ Grenada } \\
\hline Jamaica & -0.33 & -0.86 & -1.44 & -0.49 & -1.03 & -1.61 \\
\hline Kazakhstan & -0.28 & -0.43 & -0.90 & -0.19 & -0.34 & -0.81 \\
\hline Latvia & -0.24 & -0.53 & -1.31 & -0.31 & -0.60 & -1.38 \\
\hline \multicolumn{7}{|l|}{ Lebanon } \\
\hline Libya & -0.63 & -1.09 & -1.30 & -0.29 & -0.76 & -0.97 \\
\hline Lithuania & -0.45 & -0.66 & -1.55 & -0.53 & -0.74 & -1.63 \\
\hline Malaysia & -1.21 & -0.95 & -1.91 & -1.01 & -0.75 & -1.71 \\
\hline Mauritius & -0.45 & -0.32 & -0.79 & -0.54 & -0.41 & -0.88 \\
\hline Mexico & -0.23 & -0.15 & -0.31 & -0.25 & -0.17 & -0.34 \\
\hline Panama & -0.24 & -0.40 & -0.85 & -0.26 & -0.42 & -0.87 \\
\hline Poland & -0.37 & -0.68 & -1.59 & -0.43 & -0.74 & -1.65 \\
\hline Romania & -0.19 & -0.22 & -0.66 & -0.29 & -0.31 & -0.76 \\
\hline Russian Federation & -0.13 & -0.23 & -0.30 & -0.14 & -0.23 & -0.30 \\
\hline \multicolumn{7}{|l|}{ Seychelles } \\
\hline South Africa & -0.22 & -0.27 & -0.64 & -0.26 & -0.31 & -0.68 \\
\hline \multicolumn{7}{|l|}{ St. Kitts and Nevis } \\
\hline \multicolumn{7}{|l|}{ St. Lucia } \\
\hline \multicolumn{7}{|l|}{ St. Vincent \& Grens. } \\
\hline \multicolumn{7}{|l|}{ Suriname } \\
\hline Turkey & -0.24 & -0.39 & -0.69 & -0.32 & -0.47 & -0.76 \\
\hline Uruguay & -0.23 & -0.33 & -0.67 & -0.20 & -0.31 & -0.64 \\
\hline Venezuela & -0.23 & -0.53 & -0.58 & -0.23 & -0.53 & -0.57 \\
\hline Mean & -0.34 & -0.50 & -0.96 & -0.33 & -0.49 & -0.95 \\
\hline Median & -0.24 & -0.48 & -0.85 & -0.29 & -0.47 & -0.81 \\
\hline Stdev & 0.23 & 0.26 & 0.47 & 0.20 & 0.22 & 0.47 \\
\hline
\end{tabular}


Table 4 Continued

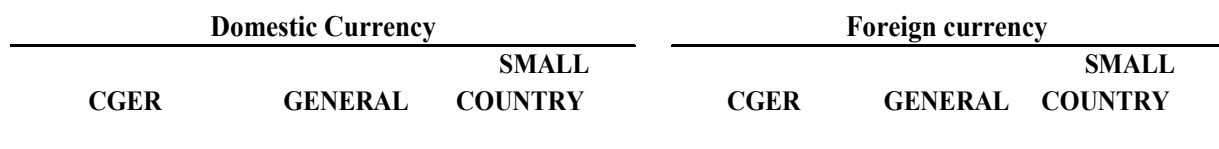

\begin{tabular}{|c|c|c|c|c|c|c|}
\hline High income: nonOECD & & & & & & \\
\hline \multicolumn{7}{|l|}{ Antigua and Barbuda } \\
\hline \multicolumn{7}{|l|}{ Bahamas, The } \\
\hline \multicolumn{7}{|l|}{ Bahrain } \\
\hline \multicolumn{7}{|l|}{ Barbados } \\
\hline \multicolumn{7}{|l|}{ Bermuda } \\
\hline \multicolumn{7}{|l|}{ Brunei } \\
\hline \multicolumn{7}{|l|}{ Cyprus } \\
\hline Estonia & -0.44 & -1.04 & -1.92 & -0.50 & -1.10 & -1.99 \\
\hline \multicolumn{7}{|l|}{ French Polynesia } \\
\hline \multicolumn{7}{|l|}{ Greenland } \\
\hline Hong Kong & -1.34 & -2.00 & -3.65 & -1.28 & -1.94 & -3.60 \\
\hline Israel & -0.30 & -0.49 & -0.60 & -0.32 & -0.50 & -0.61 \\
\hline \multicolumn{7}{|l|}{ Macau } \\
\hline \multicolumn{7}{|l|}{ Malta } \\
\hline \multicolumn{7}{|l|}{ N. Caledonia } \\
\hline Oman & -0.40 & -0.62 & -0.76 & -0.24 & -0.46 & -0.60 \\
\hline Saudi Arabia & -0.45 & -0.70 & -0.86 & -0.31 & -0.56 & -0.72 \\
\hline Singapore & -1.69 & -1.50 & -5.03 & -1.43 & -1.25 & -4.77 \\
\hline Slovenia & -0.71 & -0.72 & -3.52 & -0.73 & -0.75 & -3.55 \\
\hline \multicolumn{7}{|l|}{ Taiwan } \\
\hline Trinidad and Tobago & -0.32 & -0.67 & -1.22 & -0.26 & -0.61 & -1.15 \\
\hline Mean & -0.71 & -0.97 & -2.20 & -0.63 & -0.90 & -2.12 \\
\hline Median & -0.45 & -0.71 & -1.57 & -0.41 & -0.68 & -1.57 \\
\hline Stdev & 0.52 & 0.52 & 1.66 & 0.48 & 0.51 & 1.64 \\
\hline
\end{tabular}


Table 4 Continued

\begin{tabular}{lr}
\multicolumn{3}{c}{ Domestic Currency } \\
\hline \\
& SMALL
\end{tabular}

\begin{tabular}{ccc}
\multicolumn{3}{c}{ Foreign currency } \\
\hline CGER & GENERAL & COUNTRY
\end{tabular}

\begin{tabular}{|c|c|c|c|c|c|c|}
\hline High income: OECD & & & & & & \\
\hline Australia & -0.14 & -0.35 & -0.54 & -0.16 & -0.36 & -0.56 \\
\hline Austria & -0.44 & -0.48 & -1.43 & -0.39 & -0.44 & -1.38 \\
\hline Belgium & -0.70 & -0.39 & -2.97 & -0.68 & -0.37 & -2.94 \\
\hline Canada & -0.21 & -0.21 & -0.71 & -0.19 & -0.20 & -0.69 \\
\hline Czech Republic & -0.80 & -1.31 & -2.55 & -0.76 & -1.28 & -2.51 \\
\hline Denmark & -0.35 & -0.33 & -1.12 & -0.32 & -0.31 & -1.10 \\
\hline Finland & -0.34 & 0.05 & -0.70 & -0.30 & 0.09 & -0.66 \\
\hline France & -0.20 & -0.28 & -0.71 & -0.23 & -0.30 & -0.74 \\
\hline Germany & -0.37 & 0.03 & -1.54 & -0.32 & 0.08 & -1.50 \\
\hline Greece & -0.13 & -0.21 & -0.50 & -0.22 & -0.30 & -0.59 \\
\hline Hungary & -0.64 & -0.68 & -1.72 & -0.61 & -0.65 & -1.69 \\
\hline \multicolumn{7}{|l|}{ Iceland } \\
\hline Ireland & -0.55 & -0.24 & -1.10 & -0.41 & -0.11 & -0.96 \\
\hline Italy & -0.23 & -0.29 & -0.70 & -0.23 & -0.29 & -0.71 \\
\hline Japan & -0.12 & -0.10 & -0.47 & -0.11 & -0.10 & -0.46 \\
\hline Korea & -0.37 & -0.01 & -0.71 & -0.38 & -0.02 & -0.72 \\
\hline Luxembourg & -1.21 & -3.69 & -9.04 & -0.73 & -3.20 & -8.56 \\
\hline Netherlands & -0.57 & -0.52 & -1.62 & -0.51 & -0.45 & -1.55 \\
\hline New Zealand & -0.18 & -0.21 & -0.54 & -0.16 & -0.20 & -0.52 \\
\hline Norway & -0.38 & -0.73 & -0.97 & -0.16 & -0.51 & -0.76 \\
\hline Portugal & -0.23 & -0.49 & -1.19 & -0.28 & -0.54 & -1.23 \\
\hline Slovakia & -0.69 & -0.73 & -1.83 & -0.70 & -0.74 & -1.84 \\
\hline Spain & -0.18 & -0.36 & -0.74 & -0.24 & -0.42 & -0.80 \\
\hline Sweden & -0.37 & 0.09 & -1.10 & -0.31 & 0.14 & -1.05 \\
\hline Switzerland & -0.47 & -0.45 & -1.52 & -0.39 & -0.38 & -1.44 \\
\hline United States of America & -0.09 & -0.21 & -0.50 & -0.12 & -0.24 & -0.53 \\
\hline United Kingdom & -0.17 & -0.42 & -0.84 & -0.20 & -0.45 & -0.87 \\
\hline Mean & -0.39 & -0.48 & -1.44 & -0.35 & -0.44 & -1.40 \\
\hline Median & -0.36 & -0.34 & -1.04 & -0.31 & -0.34 & -0.92 \\
\hline Stdev & 0.26 & 0.72 & 1.67 & 0.20 & 0.63 & 1.59 \\
\hline \multicolumn{7}{|l|}{ Overall } \\
\hline Mean & -0.32 & -0.49 & -1.02 & -0.37 & -0.54 & -1.08 \\
\hline Median & -0.26 & -0.39 & -0.77 & -0.31 & -0.46 & -0.76 \\
\hline Stdev & 0.24 & 0.45 & 1.01 & 0.22 & 0.43 & 0.97 \\
\hline
\end{tabular}

Note: Income group: Economies are divided according to 2007 GNI per capita, calculated using the World Bank

Atlas method. The groups are: low income, $\$ 935$ or less; lower middle income, $\$ 936$ - \$3,705; upper middle income,

$\$ 3,706$ - \$11,455; and high income, $\$ 11,456$ or more. Source: World Bank 


\section{Price Elasticities and the Real Exchange Rate}

In the analysis described above, the behavior of exports and imports depends on prices of these goods in domestic currency terms. What is of interest, however, is how changes in the real exchange rate affects a country's trade balance. It turns out that in the context of the model laid out in section II-the three-good model of exports, imports, and nontraded goods - the real exchange can be defined as the price of traded goods relative to the price of nontraded goods. Therefore a change in the nominal price of exports or imports IS a change in the real exchange rate, holding the price of nontraded goods constant. This is done in this model because there is no formal mechanism to determine the price of nontraded goods in response to a change in the nominal exchange rate, as $P_{N}$ is endogenous. The price of nontraded goods would be determined by the interaction of both demand and supply for the nontraded good, but in the model described above, demand is not specified, as this would require the adoption of a particular functional form for the utility function.

Equation (32) gives an expression for the export supply elasticity, defined as the proportional change in export supply as a result of a proportional change in the price of exports, holding the price of nontraded goods constant. Likewise, equation (33) gives an expression for the import demand elasticity, holding the price of nontraded goods constant. Defined in these terms, these elasticities can be thought of as real exchange rate elasticities, since they both are defined holding the price of nontraded goods constant.

An alternative definition of the real exchange rate that is frequently used is based on relative CPIs of two countries - the CPI in the home country relative to the CPI in the foreign country. There are a number of issues that need to be confronted if this definition is used. First, this definition of the real exchange rate does not isolate the importance of the price of traded goods relative to nontraded goods - both types of these goods are aggregated in one price index, the consumer price index. Second, one would need to specify how the price of nontraded goods is determined. In general, a country's CPI can be thought of as a aggregation of the prices of imports, exports, and nontraded goods, along the following lines:

$$
C P I=P_{M}^{\alpha} P_{E}^{\beta} P_{N}^{1-\alpha-\beta} \text { or } \quad C \hat{P} I=\alpha \hat{P}_{M}+\beta \hat{P}_{E}+(1-\alpha-\beta) \hat{P}_{N}
$$

In equation (48), it would be straightforward to determine how the prices of imports and exports are affected by a depreciation of the home currency. However, determining how the price of nontraded goods responds is more complicated and requires a complete model because the price of nontraded goods is determined endogenously by both supply and demand. In particular, it would have to specify cross-price elasticities: how a change in the price of traded goods affects the demand for nontraded goods. In the absence of a complete model, this study does not take the approach of defining the real exchange rate as the ratio of domestic and foreign CPIs. 


\section{Some EXTENSIONS}

This section discusses how the framework described above can be adapted to include (i) imperfect competition and (ii) less-than-perfect labor mobility.

\section{A. Imperfect Competition}

The key feature of firms that are imperfectly competitive is that they determine the quantity of output that will produce based on where marginal revenue (MR) equals marginal cost (MC). As is well known, MR is always less than price. The reason for this is that in order to sell more, an imperfectly competitive firm must reduce its price, not just on the last unit sold, but on all units.

Suppose the inverse demand curve is:

$p=f(q)$

where $p$ denotes price and $q$ is quantity. Then total revenue (price times quantity) is:

$T R=f(q) q$

Marginal revenue is:

$M R=\frac{d T R}{d q}=f(q)+\frac{d f}{d q} q$, which can be written, after manipulation as:

$M R=p+\frac{p}{\eta_{d}}=p\left(1+\frac{1}{\eta_{d}}\right)$, where $\eta_{d}$ is the price elasticity of demand for the final product and $\eta_{d} \leq 0$. The term is parenthesis $\left(1+\frac{1}{\eta_{d}}\right)$ is the markup of price over marginal revenue (m). Several conclusions emerge:

- Marginal revenue is always less than price, using the above equation, since $\eta_{d}$ is negative.

- $\quad$ There is an inverse relationship between the magnitude of the markup and the price elasticity of demand. A high elasticity of demand implies a low markup and viceversa.

- $\quad$ For the size of the markup to make sense, the price elasticity of demand must be less than or equal to -1 . That is, an imperfectly competitive firm always operates on the portion of his demand curve that is price elastic. If he operated on the inelastic region, MR would be negative. 
- $\quad$ The small country assumption means that the elasticity of export demand and import supply are infinite. In light of the above, the small country assumption is incompatible with imperfect competition in the export sector. However, imperfect competition may be possible in the import and nontraded sectors.

- $\quad$ The markup, $m$ defined above, is itself an endogenous variable because it depends on the elasticity of demand, which will differ along a given demand curve.

\section{Implementing imperfect competition}

Suppose suppliers of imported intermediate inputs were monopolistic. Therefore, users of imported intermediates would pay higher prices, compared to what they would pay if markets were perfectly competitive. However, the question for those interested in trade elasticities (export supply and import demand elasticities) is not what is the impact of monopolistic pricing on elasticity values, relative to perfect competition. Rather, the question is how, if at all, does monopolistic pricing behavior affect the trade elasticities, for a given level of markups.

This is a difficult question to answer. In the context of the model described above, there is a sense in which monopolistic pricing would have no affect on the elasticity of demand for imported inputs, provided the markup remains unchanged. The problem is that the markup is itself an endogenous variable: it depends on the elasticity of demand, which varies along the demand curve. So, one would have to endogenize $m$, the markup.

\section{B. Less than Perfect Labor Mobility}

In the current setup in the model, capital is assumed to be immobile across sectors, while labor is perfectly mobile. One way to reduce the extent of labor mobility is to reduce the cost share of labor employed in a sector and increase the cost share of capital (the fixed factor).

The model described by equations (1) through (7) contains the following equations:

$$
\begin{aligned}
& \hat{w} \theta_{L E}+\hat{r}_{E} \theta_{K E}+\hat{p}_{I} \theta_{I E}=\hat{p}_{E} . \\
& \hat{w} \theta_{L M}+\hat{r}_{M} \theta_{K M}+\hat{p}_{I} \theta_{I M}=\hat{p}_{M} \\
& \hat{w} \theta_{L N}+\hat{r}_{N} \theta_{K N}+\hat{p}_{I} \theta_{I N}=\hat{p}_{N} .
\end{aligned}
$$

In this setup, capital is a sector specific factor and thus, cannot move across sectors, while labor is freely mobile. One way to reduce the degree of labor mobility would be increase the magnitude of the cost share of capital in a given sector $-\theta_{K j}$-and reduce the cost share of labor, $\theta_{L j}$. Since capital is a fixed on a sectoral basis, increasing the capital share will reduce the ability of a sector to expand in response to a price change. In effect, this will reduce the 
supply elasticity. To do this, one would need some measure of the degree of labor mobility, and correspondingly, how much the labor cost share should be reduced in each sector. Keep in mind that however the labor cost share is reduced, the sum of the labor, capital, and intermediate input cost shares must sum to one in each sector.

\section{Conclusion}

This paper presented a method for estimating import demand and export supplies on a country basis without using econometrics. The method relies on some standard results borrowed from production theory and a well-know model of international trade. In particular, using an economy's GDP function, the derivative with respect to export prices gives the export supply function (assuming all production is exported) and the derivative with respect to the price of imported intermediates gives the import demand function. This approach isolates the determinants of both the export supply and import demand functions in terms of observable data, i.e. cost and distributive shares. Using data on these determinants, this approach then permits the estimation of export supply and import demand elasticities. Econometric estimates of trade elasticities for low-income countries are particularly scarce, so one contribution of this paper is that it presents a set of elasticity values for these countries.

This paper also showed how the calculated elasticities could be used in assessing whether a country's real exchange rate is in equilibrium. It also identifies the relevant condition that must hold for a real devaluation to improve the trade balance for a "small" country, and relates this condition to the one used in exchange rate assessments for large countries. A real devaluation always improves the trade balance for a "small" country measured in foreign currency terms, but may not for a large country - one that is able to influence the international prices of its exports or imports. The last section of the paper shows how the calculation of trade elasticities might be affected by features such as imperfect competition or less than perfect mobility of labor across sectors. This paper should be useful to economists who want to calculate how a change in its real exchange rate might affect its balance of trade. 


\section{REFERENCES}

Dimaranan, Betina V. and Robert A. McDougall, 2006, Global Trade, Assistance, and Production: The GTAP 6 Data Base, Center for Global Trade Analysis, Purdue University.

Dixit, Avinash, and Victor Norman, 1980, “Theory of International Trade: A Dual General Equilibrium Approach,” (Cambridge: Cambridge University Press).

Frankel, Jeffrey, David Parsley, and Shang-Jin Wei, 2005, "Slow Pass Through Around the World: A New Import for Developing Countries?” NBER Working Paper No. 11199, (Cambridge, Massachusetts: National Bureau of Economic Research).

Jones, Ronald, 1965, “The Structure of Simple General Equilibrium Models," Journal of Political Economy, Vol. 73 (December), pp. 557-72.

Kee, Haui Looi, Alessandro Nicita, and Marcelo Olarreaga, 2008, "Import Demand Elasticities and Trade Distortions," Review of Economics and Statistics, Vol. 90, No. 4, pp. 666-682.

Khan, Mohsin, and Morris Goldstein, 1985, "Income and Price Effects in Foreign Trade," in Handbook of International Economics, volume 2, edited by Ronald Jones and Peter Kenen (New York: North Holland), pp. 1041-1105.

Kohli, Ulrich, 1991, Technology, Duality, and Foreign Trade: The GNP Function Approach to Modeling Imports and Exports (Ann Arbor: University of Michigan Press).

Senhadji, Semlali, 1997, “Time-Series of Structural Import Demand Equations-A Cross Country Analysis,” IMF Working Paper 97/132 (Washington: International Monetary Fund).

Stern, Robert, 1973, The Balance of Payments: Theory and Economic Policy, (New York: Aldine Publishing Company).

Stern, Robert, Francis, Jonathan, and Bruce Schumacher, 1976, Price Elasticities in International Trade - An Annotated Bibliography, (London: MacMillan).

Woodland, Alan, 1982, International Trade and Resource Allocation, (Amsterdam; New York: North-Holland). 
40

Appendix

The "general equilibrium" export supply elasticity is:

$$
\begin{gathered}
\hat{X}_{E}=\frac{\lambda_{L E} \sigma_{E} \theta_{K M} \theta_{K N} \theta_{I E} \sigma_{E}+\lambda_{L M} \sigma_{M} \theta_{K N}\left(1-\theta_{I M}\right) \sigma_{E}\left(1-\theta_{K E}\right)+\lambda_{L N} \sigma_{N} \theta_{K M}\left(1-\theta_{I N}\right) \sigma_{E}\left(1-\theta_{K E}\right)}{\lambda_{L E} \sigma_{E} \theta_{K M} \theta_{K N}\left(1-\theta_{I E}\right)+\lambda_{L M} \sigma_{M} \theta_{K E} \theta_{K N}\left(1-\theta_{I M}\right)+\lambda_{L N} \sigma_{N} \theta_{K E} \theta_{K M}\left(1-\theta_{I N}\right)} \hat{p}_{E} \\
+\frac{-\left[\lambda_{L E} \sigma_{E} \theta_{K M} \theta_{K N} \theta_{I E} \sigma_{E}+\lambda_{L M} \sigma_{M} \theta_{K N} \sigma_{E}\left[\theta_{I E}+\theta_{I M}\left(\theta_{K E}-1\right)\right]+\lambda_{L N} \sigma_{N} \theta_{K M} \sigma_{E}\left[\theta_{I E}+\theta_{I N}\left(\theta_{K E}-1\right)\right]\right]}{\lambda_{L E} \sigma_{E} \theta_{K M} \theta_{K N}\left(1-\theta_{I E}\right)+\lambda_{L M} \sigma_{M} \theta_{K E} \theta_{K N}\left(1-\theta_{I M}\right)+\lambda_{L N} \sigma_{N} \theta_{K E} \theta_{K M}\left(1-\theta_{I N}\right)} \hat{p}_{I}
\end{gathered}
$$

The "general equilibrium" import demand elasticity is:

$$
\begin{aligned}
& \hat{M}_{I}=\frac{1}{\left(\lambda_{L E} \sigma_{E} \theta_{K M} \theta_{K N}\left(1-\theta_{I E}\right)+\lambda_{L M} \sigma_{M} \theta_{K E} \theta_{K N}\left(1-\theta_{I M}\right)+\lambda_{L N} \sigma_{N} \theta_{K E} \theta_{K M}\left(1-\theta_{I N}\right)\right.} \\
& {\left[\begin{array}{l}
-\lambda_{I E}\left[\sigma_{E} \lambda_{L E} \sigma_{E} \theta_{K M} \theta_{K N}+\sigma_{E} \lambda_{L M} \sigma_{M} \theta_{K N}\left(1-\theta_{I M}-\theta_{L E}\right)+\sigma_{E} \lambda_{L N} \sigma_{N} \theta_{K M}\left(1-\theta_{I N}-\theta_{L E}\right)\right] \\
-\lambda_{I M}\left[\sigma_{M} \lambda_{L E} \sigma_{E} \theta_{K N}\left(1-\theta_{I E}-\theta_{L M}\right)+\sigma_{M} \lambda_{L M} \sigma_{M} \theta_{K E} \theta_{K N}+\sigma_{M} \lambda_{L N} \sigma_{N} \theta_{K E}\left(1-\theta_{I N}-\theta_{L M}\right)\right] \\
-\lambda_{I N}\left[\sigma_{N} \lambda_{L E} \sigma_{E} \theta_{K M}\left(1-\theta_{I E}-\theta_{L N}\right)+\sigma_{N} \lambda_{L M} \sigma_{M} \theta_{K E}\left(1-\theta_{I M}-\theta_{L N}\right)+\sigma_{N} \lambda_{L N} \sigma_{N} \theta_{K E} \theta_{K M}\right]
\end{array}\right] \hat{p}_{I}} \\
& + \\
& \frac{1}{\left(\lambda_{L E} \sigma_{E} \theta_{K M} \theta_{K N}\left(1-\theta_{I E}\right)+\lambda_{L M} \sigma_{M} \theta_{K E} \theta_{K N}\left(1-\theta_{I M}\right)+\lambda_{L N} \sigma_{N} \theta_{K E} \theta_{K M}\left(1-\theta_{I N}\right)\right.} \\
& {\left[\begin{array}{l}
\lambda_{I E}\left[\sigma_{E} \lambda_{L E} \sigma_{E} \theta_{K M} \theta_{K N}+\sigma_{E} \lambda_{L M} \sigma_{M} \theta_{K N}\left(1-\theta_{I M}\right)+\sigma_{E} \lambda_{L N} \sigma_{N} \theta_{K M}\left(1-\theta_{I N}\right)\right] \\
-\lambda_{I M}\left[\sigma_{M} \lambda_{L E} \sigma_{E} \theta_{K N} \theta_{L M}\right]-\lambda_{I N}\left[\sigma_{N} \lambda_{L E} \sigma_{E} \theta_{K M} \theta_{L N}\right]
\end{array}\right] \hat{p}_{E}}
\end{aligned}
$$

